\title{
NMD is required for timely cell fate transitions by fine-tuning gene expression and controlling translation
}

Elena Galimberti ${ }^{1}$, Robert Sehlke ${ }^{2 \#}$, Michelle Huth ${ }^{1 \#}$, Marius Garmhausen ${ }^{2}$, Merrit Romeike ${ }^{1}$, Julia Ramesmayer ${ }^{1}$, Sarah Stummer ${ }^{1}$, Fabian Titz-Teixeira², Veronika Herzog ${ }^{3}$, Anastasia Chugunova ${ }^{4}$, Katrin Friederike Leesch ${ }^{4}$, Laurenz Holcik ${ }^{1,6}$, Klara Weipoltshammer ${ }^{5}$, Laura Santini ${ }^{1}$, Andreas Lackner ${ }^{1}$, Arndt von Haeseler ${ }^{1,6,7}$, Christa Bücker ${ }^{1}$, Andrea Pauli ${ }^{4}$, Christian Schoefer ${ }^{5}$, Stefan L. Ameres ${ }^{3}$, Austin Smith ${ }^{8}$, Andreas Beyer ${ }^{2}$, Martin Leeb ${ }^{1 *}$

Affiliations:

${ }^{1}$ Max Perutz Labs Vienna, University of Vienna, Vienna BioCenter, Dr.-Bohr-Gasse 9, 1030 Vienna, Austria

${ }^{2}$ CECAD Cologne, Joseph-Stelzmann-Str. 2650931 Köln, Germany

${ }^{3}$ Institute of Molecular Biotechnology, Vienna BioCenter, Dr.-Bohr-Gasse 3, 1030 Vienna, Austria

${ }^{4}$ Research Institute of Molecular Pathology, Vienna BioCenter, Campus-Vienna-Biocenter 1, 1030 Vienna, Austria

${ }^{5}$ Department for Cell and Developmental Biology, Medical University of Vienna, Schwarzspanierstrasse 17, 1090 Vienna, Austria

${ }^{6}$ Center for Integrative Bioinformatics Vienna, Max Perutz Labs, University of Vienna and Medical University of Vienna, Dr.-Bohr-Gasse 9, 1030 Vienna, Austria

${ }^{7}$ Bioinformatics and Computational Biology, Faculty of Computer Science, University of Vienna, Vienna, Austria

${ }^{8}$ Wellcome - MRC Cambridge Stem Cell Institute, University of Cambridge, Cambridge CB2 OAW, UK

*corresponding author

\# equal contribution 


\section{ABSTRACT}

Cell fate transitions depend on balanced rewiring of transcription and translation programmes to mediate ordered developmental progression. Components of the nonsense-mediated mRNA decay (NMD) pathway have been implicated in regulating embryonic stem cell (ESC) differentiation, but the exact mechanism is unclear. Here we show that NMD controls the translation initiation factor Eif4a2 and its premature termination codon encoding isoform (Eif4a2 ${ }^{\text {PTC }}$. NMD deficiency leads to translation of a specific truncated Eif4a2 protein, which elicits increased translation rates and causes significant delays in mouse ESC differentiation. Thereby a previously unknown feedback loop between NMD and translation initiation is established. Our results illustrate a clear hierarchy between KOs in severity of target deregulation and differentiation phenotype (Smg5 > Smg6 > Smg7), which highlights heterodimerindependent functions for Smg5 and Smg7. Together, our findings expose an intricate link between mRNA stability and translation initiation control that must be maintained for normal dynamics of cell state transitions. 


\section{INTRODUCTION}

Mouse embryonic stem cells (ESCs) capture the developmentally transient naïve pluripotent state in vitro.

ESC self-renewal is maintained by an interactive transcription factor (TF)-network, which has been extensively characterized $^{1,2}$. This network is decommissioned between embryonic day (E)4.5 and E5.5 in mouse development ${ }^{3}$. Similar kinetics are maintained in ESCs, which irreversibly commit to differentiation 24-36 $\mathrm{h}$ after $2 \mathrm{i}$ withdrawal ${ }^{4-6}$. Rex1 is a known marker of naïve pluripotency and high-resolution dissection of the exit from naïve pluripotency is facilitated by the availability of Rex1 promoter-driven destabilized GFP reporter ESC lines (Rex1::GFPd2) ${ }^{7,8}$. Rex1-GFP downregulation is initiated within $24 \mathrm{~h}$ after $2 \mathrm{i}$ withdrawal (N24) and completed after $48 \mathrm{~h}$ (N48). Extinction of Rex1-GFPd2 expression coincides with functional commitment to differentiation.

Several genome-wide screens have uncovered drivers of the exit from pluripotency ${ }^{9}$, many of which are involved in transcriptional regulation and epigenetic modification. These screens also identified a large cohort of post-transcriptional regulators. RNA modifiers, such as $\mathrm{m} \mathrm{A}^{10,11}{ }^{11}$; negative regulators of mRNA stability, such as Pum1 ${ }^{12}$; and components of the nonsense-mediated mRNA decay (NMD) pathway ${ }^{12-14}$ have been implicated in regulating ESC differentiation. Nonetheless, how post-transcriptional regulatory mechanisms contribute to cell fate changes remains poorly understood.

NMD is a translation coupled mechanism that promotes degradation of mRNAs containing a premature termination codon (PTC) ${ }^{15}$. However, PTC-independent NMD activity has also been shown ${ }^{16-18}$. NMD is triggered by phosphorylation of the RNA-helicase Upf1, which is essential for NMD. Degradation occurs either by Smg6-mediated endonucleolytic cleavage, or by exonucleolytic cleavage, mediated by a Smg5Smg7 heterodimer ${ }^{15}$. Transcriptome-wide analysis demonstrated that Smg6 and Smg5-Smg7 have highly overlapping mRNA targets ${ }^{19}$. There is also evidence that Smg factors regulate telomere maintenance ${ }^{20}$ ${ }^{22}$. Although NMD components constitute some of the top hits in genome-wide screens studying exit from 
naïve pluripotency, neither the contribution of individual NMD effector proteins, nor the key mRNA targets of NMD that lead to delayed cell fate transition are known.

Here we identify a role for NMD in ensuring normal differentiation kinetics by facilitating establishment of proper cell fate specific gene expression programmes and by controlling expression of Eif4a2, a key translation initiation factor. We identify the resulting Eif4a2-dependent increased translational activity in NMD-deficient ESCs as the main reason for their inability to properly commit to differentiation.

\section{RESULTS}

\section{Variable degree of defects in exit from naïve pluripotency in NMD-deficient ESCs}

To delineate the molecular function of NMD in the exit from naïve pluripotency, we generated Rex1GFPd2 reporter ESC lines ${ }^{4,23}$ deficient for the three NMD downstream effectors $5 m g 5$, Smg6 or Smg7 (NMD KO ESCs), and corresponding rescue cell lines in which the missing NMD-factor was re-expressed (NMD rescue ESCs) (Extended Data Fig. 1a,b). NMD KO cells showed normal ESC morphology, cell cycle profile and telomere maintenance (Extended Data Fig. 1c,d), but exhibited pronounced delays in the exit from naïve pluripotency. This manifested in delayed downregulation of the Rex1-GFPd2 reporter $24 \mathrm{~h}$ after the onset of differentiation (N24) and delayed entry into commitment, assayed $72 \mathrm{~h}$ after $2 \mathrm{i}$ withdrawal (Fig. 1a,b). Both defects were restored in rescue cell lines, showing causality of NMD-factor deficiency for delays in exit from naïve pluripotency (Fig 1a,b). The three NMD-factor KOs displayed variable degrees of differentiation delays: the strongest effect was observed in the absence of Smg5 and the weakest in the absence of Smg7. This does not align with the proposed strictly heterodimerdependent function of Smg5 and Smg7, which would predict similar phenotypes for Smg5 and Smg7 KOs 24-26. The results therefore suggest a less strict heterodimer dependence than anticipated for the activity of either Smg5 or Smg7. 
47 To monitor the effect of NMD deficiency on long-term differentiation potential, we performed 3D aggregate embryoid body (EB) differentiation during a 10-day time-course. As expected, downregulation of naïve and primed pluripotency markers was severely impaired in NMD KO EBs. Although NMD KO aggregates upregulated the formative marker genes Fgf5 and Otx2 with similar kinetics to WT, the subsequent shutdown of the formative program, observed in WT cells between day 4 (d4) and d6, was impaired (Fig. 1C). Furthermore, expression of the endo-mesoderm defining TF Brachyury $(T)$ was not properly downregulated after a peak of expression at $d 4$ to $d 6$, suggesting a general function of NMD in shaping transcriptomes during early cell fate decisions (Fig. 1C). Further supporting a role for NMD in regulating differentiation, teratomas derived from Smg6 KO ESCs showed a lower degree of differentiation than WT and rescue controls (Extended Data Fig. 1e). Together, these data show a global differentiation defect of Smg factor-deficient cells, with a variable degree of phenotypic strengths (Smg5 $>$ Smg6 > Smg7).

\section{Cooperativity between NMD factors regulates NMD and exit from naïve pluripotency}

To investigate potential cooperativity between NMD effectors, we performed siRNA-mediated knockdowns of Smg6 and Smg7 in Smg5 and Smg6 KO ESCs (Fig. 1d-h and Extended Data Fig. 1f). Thereby, we generated cells double- and triple-depleted for NMD downstream effectors. WT cells showed a near complete loss of Rex1-GFPd2 expression at N48 (Fig. 1d). siRNA-mediated depletion of Smg6 in Smg5 KO cells resulted in only a minor, non-synergistic increase in the differentiation delay assessed in Rex1-GFPd2 reporter assays (Fig. 1e), despite the strong defects observed in both single KOs. By contrast, the depletion of Smg7 in a Smg6 KO background yielded a strong synergistic differentiation phenotype (Fig. 1f). Similarly, co-depletion of both heterodimer members, $S m g 5$ and Smg7, exhibited a clear synergistic effect (Fig. 1g), further highlighting heterodimer-independent functions for Smg5 and Smg7. Combined 
depletion of all the NMD effectors by double knockdown of Smg6 and Smg7 in Smg5 KO ESCs resulted in differentiation delays on par with $S m g 6 / 7$ or $5 m g 5 / 7$ double depletion (Fig. 1h). To assess the impact of NMD effector depletion and co-depletion on NMD-specific mRNA target gene expression, we assessed levels of the known NMD target gene Gadd45b by RT-qPCR ${ }^{16}$. Consistent with observed differentiation delays, we observed strong genetic interactions between Smg6 and Smg7 as well as between Smg5 and Smg7, and weaker interactions between Smg5 and Smg6 regarding Gadd45b transcript abundance (Extended Data Fig. 1g). Together, this suggests that differentiation delays scale with the extent of NMD impairment.

To further study the cooperativity of NMD factors we sought to generate stable NMD double KO (dKO) ESCs. However, attempts using CRISPR/Cas9 to generate all possible dKO ESC lines yielded only Smg5/Smg6 (Smg5/6) dKO ESCs. Neither Smg5/Smg7 nor Smg6/Smg7 dKOs could be established, despite multiple attempts using efficient gRNAs. This suggests an essential role of Smg7 in ESCs in the absence of its heterodimerization partner Smg5 or in the absence of Smg6. Smg5/6 dKO cells showed a deficiency in downregulating naïve TF mRNAs similar to that observed in Smg5 single KO cells, indicating a dominant role of Smg5 in regulating differentiation-relevant RNA-homeostasis programmes (Fig. 1i).

Taken together, Smg5, Smg6 and Smg7 KO ESCs exhibit variable degrees of differentiation defects and NMD downstream factors act synergistically during the exit from pluripotency. Smg7 possesses a central, facultatively essential function: It can be depleted without resulting in strong effects on early differentiation, but an essential role is revealed in the absence of either Smg5 or Smg6.

\section{Smg7 is necessary and sufficient for pUpf1 binding, independently of Smg5}

Continuous NMD activity relies on cyclic phosphorylation and dephosphorylation of Upf1. We observed increased pUpf1 levels in all NMD KO cells (Fig. 2a), in line with previous reports ${ }^{25}$. Notably, pUpf1 levels 
followed the same trend as the differentiation phenotype and deregulation of the NMD target Gadd45b (pUpf1 levels in Smg5 KO > Smg6 KO > Smg7 KO > WT ESCs). Upf1 is phosphorylated by the kinase Smg1 27, 28. Smg1 mRNA is itself an NMD target, but in contrast to pUpf1 is upregulated to a similar extent in all three NMD KO ESCs (Extended Data Fig. 2a). Therefore, graded increase of pUpf1 levels in NMD KOs is unlikely to be a direct effect of higher Smg1 expression. Impaired Upf1 dephosphorylation may therefore underlie increased pUpf1 levels in NMD mutant cells. The three NMD effectors Smg5, Smg6 and Smg7 have all been implicated in the recruitment of PP2A to dephosphorylate Upf1 ${ }^{25,26}$, but the strongest increase of pUpf1 in Smg5 KO ESCs suggests that Smg5 acts as the main PP2A recruiter during NMD (Extended Data Fig. 2b).

To identify the molecular basis for an apparent heterodimer-independent function of $\$ m g 5$ and $S m g 7$ and for the observed genetic interactions between Smg factors, we performed co-immunoprecipitation (coIP) experiments. We precipitated Smg5 or Smg7 in WT and NMD KO ESCs, in the presence and absence of RNA. Smg5 and pUpf1, but not Smg6, co-immunoprecipitated with Smg7 in an RNA-independent manner in WT ESCs (Fig. 2b). Interestingly, Smg7 efficiently bound pUpf1 in the absence of its heterodimerization partner Smg5 (Fig. 2b). The Smg7-pUpf1 interaction was also maintained in Smg5/6 dKO ESCs, suggesting that Smg7 can interact with pUpf1 independently of other downstream NMD effectors. Conversely, however, Smg5 failed to bind pUfp1 in the absence of Smg7 (Fig. 2c), indicating that Smg7 is the critical docking site for pUpf1 during NMD and that pUpf1 binding to Smg7 is independent of Smg5. When we performed the same co-IP experiments in the presence of RNA, we observed an interaction between Smg5 or Smg7 and Smg6 in the absence of the respective heterodimerization partner (Extended Data Fig. 2c,d). This RNA-dependent interaction was not observed in WT (Fig. 2b,c).

Taken together, biochemical analysis and viability of Smg5/6 dKO ESCs indicate that Smg7 can bind to pUpf1 in the absence of Smg5 to sustain limited NMD function. Smg5, however, is unable to interact with pUpf1 in the absence of Smg7 (Fig. 2c and Extended Data Fig. 2d). The strong differentiation phenotype 
117 of Smg5-deficient cells can be explained by efficient binding of pUpf1 by Smg7 alone and subsequent

118 inefficient dephosphorylation and target degradation, resulting in a 'poisoning' of both branches of NMD

119 (Fig. 2d). By contrast, deficiency in Smg7 results in failure to recruit components of the exonucleolytic

120 branch, but allows potential recognition and degradation by Smg6, resulting in only a mild NMD target

121 deregulation and differentiation phenotype.

To uncover the molecular mode of action by which NMD regulates the exit from naïve pluripotency, we applied a combinatorial approach based on the following logic: an NMD target transcript relevant in the context of differentiation must show elevated expression levels, following the same graded pattern as the differentiation phenotype (Smg5 > Smg6 > Smg7) and a concomitant increased mRNA half-life. h of differentiation (N24) to assess the impact of NMD factor depletion on global gene expression (Fig. 3ad and Extended Data Table 1). In 2i, we identified 881 transcripts deregulated in NMD KO ESCs compared to WT (adj. $p \leq 0.01), 516$ of which were significantly upregulated and 252 significantly downregulated in all three NMD KO ESCs. This shows a strong overlap in deregulated genes between all three KO cell lines; only 113 transcripts did not show the same directionality in transcriptional changes between all NMD KO cells (Fig. 3a). Overall, upregulated transcripts (clusters 2 and 4), showed a graded increase in deregulation (Smg5 KO > Smg6 KO > Smg7 KO), similar to the KO phenotypes (Fig. 3b). 256 out of 564 transcripts belonging to clusters 2 and 4 were significantly upregulated in all three NMD KO cell lines and showed a graded response to Smg-factor depletion, suggesting that genes found within this group are responsible for the observed differentiation delays. 
GO analysis of the 256 graded upregulated transcripts in $2 \mathrm{i}$ revealed an enrichment for methyltransferase

141 and helicase activity. The remaining upregulated transcripts in $2 \mathrm{i}$ showed enrichment for telomere

142 maintenance and helicase activity. Within downregulated genes we detected an enrichment for

143 transcripts involved in neural development, somite development and other differentiation related

144 processes (Extended Data Fig. 3a and Extended Data Table 2). This suggests that leaky expression of the differentiation programme in $2 \mathrm{i}$ is dampened in NMD-deficient cells.

At N24, we identified 1,174 deregulated transcripts in NMD KO cells compared to WT cells (adj. $p \leq 0.01$ ).

Among these, 727 were upregulated in all NMD KO cells and 336 were downregulated in all NMD KO cells

(Fig. 3c). Out of the 808 upregulated transcripts belonging to clusters 2 and 3, 434 transcripts were upregulated following the graded pattern ( $\mathrm{Smg} 5 \mathrm{KO}>\mathrm{Smg} 6 \mathrm{KO}>\mathrm{Smg} 7 \mathrm{KO}$ ) (Fig. 3d). GO analysis of these 434 transcripts revealed enrichment for pluripotency-related terms, such as LIF response and stem cell maintenance (Extended Data Fig. 3b and Extended Data Table 2). This was not evident for the 293 transcripts not showing graded upregulation. Within downregulated transcripts, we detected an enrichment of differentiation-related terms, such as neural tube development and pattern specification

154 (Extended Data Fig. 3b and Extended Data Table 2). This suggested that genes showing graded expression are involved in and reflect the different levels of differentiation delays seen in NMD KO cells.

Previous reports showed increased c-Myc levels in NMD-deficient ESCs and proposed a causative 157 involvement of c-Myc in NMD KO induced differentiation delays ${ }^{13}$. However, we did not detect increased c-Myc transcript levels by RNA-seq or protein levels on western blots in any of the three NMD KO ESCS observed in NMD KO ESCs. 
Elevated transcript levels of direct NMD targets are expected to result from increased half-lives, based on impairment of mRNA degradation. Therefore, we used SLAM-seq to measure transcriptome-wide halflives ${ }^{29}$. We detected a global increase of transcript half-lives in Smg5 and Smg6 KO ESCs compared to WT ESCs (Fig. 3e). We were able to calculate half-lives for 4,342 transcripts; 3,062 of which exhibited a longer half-life in NMD KO cells (Fig. 3e). On average, mRNA half-life was significantly longer in NMD KOs than in WT $\left(\mathrm{p}<10^{-4}\right)$, increasing from $2.1 \mathrm{~h}$ in WT to 2.7 and $3 \mathrm{~h}$ in Smg5 KO and Smg6 KO, respectively (Fig. 3e). As a cohort, pluripotency TF-encoding mRNAs exhibited a significant increase in half-lives (Fig. 3f) similar to the overall increase of half-lives across the entire transcriptome. Both identity of transcripts and amplitude of half-life changes showed a strong overlap between Smg5 and Smg6 KO ESCs (Extended Data Fig. 3g). Transcriptome-wide comparisons showed that the vast majority of transcriptionally upregulated genes showed a concomitant increase in half-lives (Fig. 3g and Extended Data Fig. 3h).

To identify bona-fide NMD targets with relevance for the differentiation delay phenotype, we investigated a group of 250 genes that were significantly upregulated in all three Smg KO ESCs in $2 \mathrm{i}$ for which we could also calculate half-lives. Of these, 136 showed an increase in half-lives in both Smg5 and Smg6 KOs, and 57 showed an expression pattern in accord with the degree of phenotype-strength (Fig. 3h, Extended Data Fig. 3i and Extended Data Table 3). These 57 genes were involved in various cellular processes, such as tRNA modification, TOR-signaling and calcium transport (Extended Data Table 3). None of the naïve pluripotency factors was part of this group, suggesting that direct regulation of naïve pluripotency factors by NMD is not the primary cause for the observed differentiation delays in NMD KO ESCs. Taken together, we identified a cohort of 57 direct NMD target genes, with an expression pattern suggestive of an involvement in controlling the exit from naïve pluripotency. 

helicase Eif4a2, a component of the Eif4F translation initiation complex, which mediates 5' cap recognition by the small ribosomal subunit ${ }^{30,31}$. Several layers of evidence suggest Eif4a2 as a bona-fide NMD target. Firstly, the Eif4a2 locus encodes for two splice-isoforms: one full-length protein, and one PTC-containing isoform (Fig. 4a). Secondly, Eif4a2 mRNA levels were significantly increased after translation inhibition by cycloheximide (CHX) treatment, a hallmark of bona-fide NMD targets (Fig. 4b). CHX response was similar to that observed for Gadd45b. The PTC-isoform (Eif4a2 ${ }^{\text {PTC }}$ ) showed an even more pronounced sensitivity to $\mathrm{CHX}$ treatment. Thirdly, Eif4a2 ${ }^{\text {PTC }}$ transcript levels were upregulated in a graded fashion (Smg5 $\mathrm{KO}>$ Smg6 KO > Smg7 KO) throughout a long-term EB differentiation time-course, consistent with phenotypic relevance (Fig. 4c). Full length Eif4a2 (Eif4a2 $2^{\mathrm{FL}}$ ) protein levels were increased in NMD KO cells (Fig. 4d). The Eif4a2 ${ }^{\text {PTC }}$ isoform produced a protein that was weakly detected by western blot analysis in WT and NMD rescue cells and strongly increased in all three NMD KO ESCs, both in $2 \mathrm{i}$ and at N24 (Fig. 4d and Extended Data Fig. 4a). This indicates that while expression levels are tightly regulated by NMD, the Eif4a2 ${ }^{\text {PTC }}$ protein shows low levels of expression in WT cells. The upregulation of Eif4a2 was unique among Eif4F complex members, since the others, including the close homologue Eif4a1, showed neither increased expression nor significantly longer half-lives in NMD KOs (Fig. 4d, Extended Data Fig. 4b,c and Table S3), nor did they react to $\mathrm{CHX}$ treatment (Extended Data Fig. 4d). Taken together, this identifies Eif4a2 as a bona-fide NMD target in ESCs and shows that NMD disruption leads to the production of an Eif4a2 ${ }^{P T C}$ protein.

The linkage of NMD to Eif4a2 expression appears to be conserved in evolution. The gene structure of Eif4a2 is strikingly similar between mouse, dog and human (Fig. 4e). The mouse Eif4a2 coding sequence showed identities of $94 \%, 95 \%$ and $98.5 \%$ to human, dog and rat, respectively (Fig. 4f). Despite the PTCexon being under no apparent selective pressure to maintain protein-coding potential (PTC at nucleotide position 8 out of 107 in this exon), we observed $96.4 \%, 98.2 \%$ and $99 \%$ nucleotide identity for human, dog 
and rat, respectively, on par with the other well conserved Eif4a2 exons. Conservation of UTRs and introns was significantly lower (Fig. 4f). In a transcriptome-wide comparison, the Eif4a2 PTC exon showed high conservation at the nucleotide level: less than $5 \%$ of all exons and less than $3 \%$ of all UTRs showed higher conservation rates between mouse and rat than the Eif4a2 $2^{\text {PTC }}$ exon (Fig. 4g and Extended Data Fig. 4e-g). In the comparisons with human and dog these percentages were even lower. Therefore, the potential of Eif4a2 to be regulated by NMD is a feature conserved in mammalian evolution.

\section{Eif4a2 is causative for defects in exit from naïve pluripotency in NMD-deficient ESCs}

To delineate a potential causative relationship between increased Eif4a2 levels and the differentiation defect observed in NMD-deficient cells, we generated Eif4a2 KO cells and NMD (Smg5 or Smg6) / Eif4a2 double deficient cells (collectively referred to as NMD/Eif4a2 $\mathrm{dKO}$ ) by deleting all potential Eif4a2 isoforms or specifically Eif4a2 ${ }^{\text {PTC }}$ (Fig. 5a and Extended Data Fig. 5a). All Eif4a2 KO cell lines proliferated normally, showed normal ESC morphology and could be maintained for more than 10 passages. Deletion of the PTC exon resulted in an increase in full length Eif4a2 protein levels in WT and NMD KO ESCs (Fig. 5a). Both the complete absence of Eif4a2 or specific deletion of Eif4a2 $2^{P T C}$ accelerated Rex1-GFPd2 downregulation kinetics in WT cells (Fig. 5b), suggesting that increased expression of Eif4a2 ${ }^{\text {PTC }}$ and not full-length Eif4a2 is the major cause of the differentiation block. In NMD/Eif4a2 ${ }^{F L}$ and NMD/Eif4a2 ${ }^{P T C}$ dKO cells, differentiation kinetics were substantially rescued in a Rex1-GFPd2 assay (Fig. 5b). Accordingly, KIf4 and Nanog expression levels were downregulated to near WT-levels at N30 (Fig. 5c). Similar effects were observed for protein levels of the naïve TFs Nanog and Esrrb at N24 (Extended Data Fig. 5b). Phenotypic rescue in $\mathrm{NMD} /$ Eif4a2 $2^{\text {PTC }}$ double KO cells, in which Eif4a2 ${ }^{\mathrm{FL}}$ protein levels are even further increased, supports the proposition that Eif4a $2^{\text {PTC }}$ is the major mediator of the observed defect in exit from naïve pluripotency. 
To assess the specificity of Eif4a2 depletion in restoring differentiation potential in an NMD-deficient ESC background, we compared differentiation potential between Tcf7l1 KO and Eif4a2/Tcf7l1 dKO ESCs. Tcf7l1 encodes for a HMG box transcription factor and is one of the strongest drivers of the exit from naïve pluripotency. Tcf7l1 KO ESCs showed defects in exit from naïve pluripotency on par with NMD KOs, but exhibited an NMD-independent, Gsk3 inhibition-like phenotype (Extended Data Fig. 5c) ${ }^{4}$. Tcf7l1 KO cells were insensitive to co-depletion of Eif4a2 (Extended Data Fig. 5d), suggesting that Eif4a2 is a specific genetic interactor of NMD in regulating differentiation.

To test whether Eif4a2 upregulation is sufficient to cause a differentiation delay, we overexpressed flag-

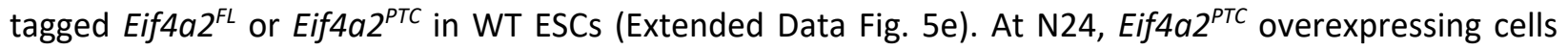
showed increased transcript levels for Klf4, Esrrb, Tfcp2/1 and Nanog and increased protein levels for Nanog at N24 (Fig. 5d,e). Cells overexpressing Eif4a2 ${ }^{F L}$ exhibited a weaker effect and showed mild upregulation only of Nanog. This accords with increase of Eif4a2 ${ }^{\mathrm{PTC}}$ rather than Eif4a2 $2^{F L}$ having a major impact on the exit from naïve pluripotency. Notably, the differentiation delays elicited by increasing expression levels of Eif4a2 isoforms did not reach the intensity observed in NMD KO ESCs, suggesting that factors in addition to Eif4a2 contribute to the failure to properly shut down naïve pluripotency in NMD KO ESCs. However, double depletion experiments show that deregulation of Eif4a2 is the major cause for exit from naïve pluripotency defects.

To test whether upregulation of Eif4a2 $2^{\text {PTC }}$ mRNA or Eif4a $2^{\text {PTC }}$ protein was responsible for the delay in differentiation of NMD KO ESCs, we introduced a frameshift mutation in the first exon of Eif4a2 ${ }^{\text {PTC }}$ (Eif4a2 ${ }^{\text {PTC-mut }}$ (Extended Data Fig. 5f). This mutation does not allow the generation of a functional Eif4a2

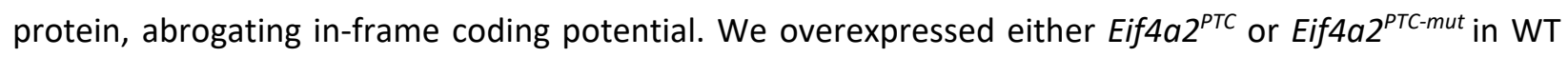
ESCs (Extended Data Fig. 5g). At N16, cells overexpressing Eif4a2 ${ }^{\text {PTC }}$ showed upregulation of Nanog and KIf4 mRNA levels, while cells overexpressing Eif4a2 ${ }^{\text {PTC-mut }}$ did not (Fig. 5f). This suggests that upregulation of the truncated protein encoded by Eif $4 a 2^{P T C}$ is responsible for the differentiation delay observed in NMD 
KO cells. In summary, these data show that Eif4a2 is the NMD target causative for the observed differentiation delays in Smg-factor deficient ES cells.

Since Eif4a2 functions as a translation initiation factor, we tested whether its upregulation in NMD KO cells resulted in increased translation rates. To this end we performed radioactive $\mathrm{S}^{35}$ and $\mathrm{O}$-propargylpuromycin (OPP) based translation assays and detected an increase in translation rates in NMD-deficient cells in $2 \mathrm{i}$ and at N12 (Fig. 6a-c and Extended Data Fig. 6a). The increase in translation rates observed in NMD KO ESCs was similar in magnitude to that observed for Tsc2 KOs, in which deregulated mTORC1 activity leads to an increase in translation ${ }^{32}$. Full Eif4a2 KOs, but also specific depletion of the PTC isoform resulted in mild reductions of translation rates. Eif4a $2^{\mathrm{FL}}$ and Eif4a $2^{\mathrm{PTC}}$ depletion in NMD KO cells reduced translation rates to WT levels, showing that increased translation rates in NMD KO cells are dependent on the PTC isoform of Eif4a2 (Fig. 6b,c).

To identify transcripts whose translation was potentially most affected by Eif4a2, we performed RIP-Seq ${ }^{33}$, using Eif4a2 KO ESCs engineered to only express either flag-tagged Eif4a2 ${ }^{\mathrm{PTC}}$ or Eif4a2 ${ }^{\mathrm{FL}}$ (Extended Data Fig. 6b). As expected for a translation factor, noncoding transcripts like Meg3 were depleted in flagEif4a2 $2^{\mathrm{FL}}$ RIP samples, indicating the specificity of the assay. Out of 9,812 expressed transcripts (CPM $>1$ in all input samples), we detected 362 transcripts significantly bound by Eif4a2 (Extended Data Table 4).

274 Several naïve pluripotency (Esrrb, Tbx3, and KIf4) and exit-factor (Jarid2, Alg13 and Fbxw7) ${ }^{6}$ encoding transcripts were enriched for Eif4a2 binding, as confirmed by RIP-qPCR (Fig. 6d and Extended Data Fig 6c). RIP-seq of flag-Eif4a2 $2^{\text {PTC }}$ resulted in only weak enrichments, possibly owing to the unstable nature of the 277 Eif4a2 ${ }^{\text {PTC }}$ protein. RIP-RT-qPCR could not detect binding of Eif4a2 ${ }^{\text {PTC }}$ to Eif4a2 ${ }^{\mathrm{FL}}$ targets identified above 278 (Extended Data Fig. 6d). 
To understand the interaction landscape of both Eif4a2 isoforms, we used KO cells expressing either flagtagged Eif4a2 ${ }^{\mathrm{PTC}}$ or Eif4a2 ${ }^{\mathrm{FL}}$ (Extended Data Fig. 6b). We also over-expressed Eif4a2 ${ }^{\mathrm{PTC}}$ in WT ESCs to identify potential Eif4a2 $2^{\mathrm{FL}}$-dependent interactions of Eif4a $2^{\mathrm{PTC}}$. We then performed co-IP followed by mass-spectrometry to identify interaction partners of the two different isoforms. To compensate for the highly unstable nature of Eif4a2 $2^{\mathrm{PTC}}$, we performed sample preparation after short-time treatment with the irreversible proteasome inhibitor epoxomicin ${ }^{34}$. Thereby, we identified 254 and 306 proteins specifically bound by Eif4a2 $2^{\mathrm{FL}}$ or Eif4a2 $2^{\mathrm{PTC}}$ over flag-only control IPs, respectively (Extended Data Table 5 and Fig. 6e). Among these, 75 proteins precipitated with both isoforms. Twenty-one of these showed near equal interactions between the Eif4a2 isoforms, including the NMD factor Smg7 and the translation initiation factor transporter Eif4enif1 (Extended Data Table 5), suggesting some preservation of normal interaction profiles of the PTC-specific isoform. Of the remainder, 47 showed stronger binding by Eif4a2 $2^{\mathrm{FL}}$. Among these were several negative regulators of mRNA stability and regulation (e.g., Ago2, Igfbp1 and Igfbp3, Pum1, Trim71, Cnot). We further detected physical association of Eif4a $2^{\mathrm{FL}}$ with several NMD components, such as Upf1 and Smg6, consistent with previous reports ${ }^{35}$. Together, this indicates an intricate link between Eif4a2 and the mRNA destabilization machinery. We detected seven factors that showed stronger co-precipitation with the PTC-isoform. These include the pluripotency TF NrOb1 (Dax1) ${ }^{8}$, the pluripotency regulator $\mathrm{Ogt}^{36}$ and the GATOR complex member and mTOR regulator Wdr59 (Fig. $6 f$ ).

Among the 178 proteins specifically bound to Eif4a $2^{\mathrm{FL}}$ and not to the PTC isoform were various translation initiation factors including all Eif4G isoforms and several components of the Eif3 complex (Fig. 6g and Extended Data Table 5). This highlights the integration of Eif4a ${ }^{\mathrm{FL}}$ (but not Eif4a2 ${ }^{\mathrm{PTC}}$ ) into a functional translation initiation complex. Eif4a1 was not detected, indicating a mutually exclusive presence in the complex. Notably, Sox2 interacted with Eif4a $2^{\mathrm{FL}}$ (Fig. 6g), indicating a potentially functional interaction of core pluripotency regulators and the translation initiation machinery. 
Among the 231 proteins specifically associated with the PTC isoform and not the full-length protein, we detected the Fgf/ERK pathway component Raf1, the mTORC1 regulator Tsc2 and the GATOR complex member Mios (Fig. 6h and Extended Data Table 5). Increased mTORC1 activity in NMD is evident by increased p-p70-S6K levels in NMD KO cells (Extended Data Fig. 6e). We further detected an interaction of Eif4a2 $2^{\text {PTC }}$ with the key pluripotency factor Oct4 (Pou5f1).

Taken together, these results indicate that Eif4a2 $2^{\mathrm{FL}}$ is mainly involved in translation initiation, but shows significant interactions with a set of mRNA destabilizing proteins, including NMD-factors, CCR4-CNOT components and the RISC member Ago2. The unstable Eif4a2 ${ }^{\text {PTC }}$ protein showed little association with translation initiation complex members, but still binds mRNA binding proteins, including the NMD factors Smg6 and Smg7.

In summary, we show that NMD controls transcript levels of hundreds of genes in ESCs and during early differentiation. This results in delayed exit from naïve pluripotency and a failure to shut down cell fate programmes in later cell fate decisions. Deregulation of a feedback circuit between NMD and translation initiation in NMD mutant cells, encoded in a PTC-containing isoform of Eif4a2, results in elevated translation rates, which are the major cause of NMD-associated differentiation defects in ESCs.

\section{DISCUSSION}

Here we report a function of NMD in the dynamic regulation of a mammalian cell state transition, the exit from naïve pluripotency. Our results highlight a role for NMD far beyond purging PTC containing transcripts. We here show a major role in modulating gene expression profiles and also translation, suggesting NMD as important component of the cellular machinery maintaining cell identity and proper differentiation trajectories.

Without NMD function, increased levels of Eif4a2 and Eif4a2 ${ }^{\text {PTC }}$ disrupt normal differentiation kinetics and lead to an increase in translation levels, including those of naïve pluripotency TFs, which impedes 
developmental transition. Basal leaky expression of differentiation-associated transcripts was reduced already in 2i. Consistently, NMD depletion leads to increased similarities between ESCs and the naïve in vivo epiblast ${ }^{6}$. Therefore, NMD acts already in self-renewal conditions to prune the differentiation programme. Despite exhibiting delayed downregulation of the pluripotency TF-network, NMD KO EBs eventually initiate formative and primed expression programmes. However, subsequent downregulation of key markers of these states, normally observed around d4 to d6 of EB differentiation, does not occur. This suggests a general role for NMD in dynamically shaping and fine-tuning transcript abundance to facilitate rapid gene regulatory network remodeling during cell fate decisions.

Differentiation defects across Smg5, Smg6 and Smg7 KOs scale with deregulation of known NMD target genes and levels of phospho-Upf1, suggesting that NMD defects directly translate to differentiation delays. The striking difference in NMD target regulation and extent of differentiation delays between Smg5 and Smg7 KOs (Smg5 >> Smg7) together with the strong synergistic effects after co-depletion are difficult to reconcile with the proposed obligatory heterodimer dependence of these two factors ${ }^{24,26}$. We show that a division of labor between $\mathrm{Smg} 5$ and $\mathrm{Smg} 7$ is causative for the different effects of depletion in the respective KOs. Smg factors have a dual role in triggering RNA degradation and in mediating Upf1 dephosphorylation; pUpf1 increase in all three Smg KOs accords with such a role of Smg factors in PP2A recruitment ${ }^{25,26}$. Our data suggest that Smg7 acts as the main adapter or recruiter for pUpf1. However, by itself it is unable to efficiently dephosphorylate Upf1, as evidenced by high pUpf1 levels in Smg5 KO ESCs, where Smg7 binding to pUpf1 is unaffected. We propose that Smg7 binding to Upf1 without subsequent Upf1 dephosphorylation results in jamming of the dephosphorylation cycle, and ensuing stalling of the mRNA degradation circuit. Aberrant recruitment of Smg6 to mRNA targets already bound by Smg7 and pUpf1 cannot properly restore NMD function in Smg5 KOs. Smg5 alone is unable to bind pUpf1 in our assays. This suggests that the main role of Smg5 lies in its dephosphorylation activity, but 
recruitment to its substrate is Smg7-dependent. This is consistent with the strongest increase of pUpf1 levels and consequently the strongest differentiation delays in Smg5 KO ESCs.

Absence of Smg6 causes a strong differentiation delay. This suggests that the exonucleolytic pathway cannot completely compensate for loss of the endonucleolytic activity in Smg6 KOs. Smg7 KO leads to very weak phenotypes and low levels of NMD deregulation. Our data support a model in which, in the absence of Smg7, Smg5 cannot be recruited to pUpf1-bound mRNAs, enabling Smg6 to recognize these potential targets and initiate their degradation. Thereby Smg6 can largely compensate for loss of Smg7, resulting in only mild NMD defects in Smg7 KO cells. However, Smg6 is unable to compensate for the complete absence of the exonucleolytic branch of NMD, suggested by the apparent loss of viability in a Smg5/7 dKO situation. This indicates a function of Smg5 and/or Smg7 in the endonucleolytic decay pathway. Our data are consistent with a function of Smg5 or Smg7 in Upf1 dephosphorylation, which is a key step also in the endonucleolytic Smg6-mediated mRNA decay axis. Despite the weak defects in single Smg7 KO cells, facultative essentiality for $S m g 7$ is revealed in the absence of either Smg5 or Smg6. Accordingly, only Smg5/Smg6 dKO ESCs could be established, showing that Smg7 is sufficient to sustain minimal NMD activity required for survival. This suggests that Smg7 can either interact with potentially novel interactors in the absence of its normal interaction partners, or that Smg7 acts as a jack of all trades, fulfilling minimal roles in target recognition, recruitment of the degradation machinery and Upf1 dephosphorylation. Taken together, the mode of action described above explains both the graded pUpf1 levels as well as graded impact on NMD of Smg5, Smg6 and Smg7 KOs.

A combination of RNA-seq and SLAM-seq based half-life analyses allowed us to compile a list of highconfidence NMD targets during the exit from pluripotency. Our data show partial redundancy and highly overlapping target gene sets between NMD downstream effectors in ESCs, consistent with previous results showing shared NMD targets between Smg6 and Smg ${ }^{19}$. However, while target mRNA identity 
appears identical, the amplitude of mRNA deregulation scales with both the phenotype and the increase in pUpf1 levels.

NMD targets showing a graded upregulation pattern (Smg5 > Smg6 > Smg7) include the translation initiation factor Eif4a2. In contrast to previous reports, c-myc was not identified as an NMD target and showed no relevance for the differentiation defect in our experiments ${ }^{13}$. We believe that our use of stateof-the-art ESC culture conditions facilitated the identification of differentiation-relevant NMD targets, that are obscured in heterogeneous FCS/LIF culture conditions.

In mammals, two homologs of the Eif4a helicase, Eif4a1 and Eif4a2, can be integrated into the elF4F complex ${ }^{30,31}$. In our datasets only Eif4a2, and not Eif4a1, was found to be regulated by NMD. In addition to encoding for a full-length protein, the Eif4a2 locus also produces a distinct PTC-containing isoform. In the truncated Eif4a2 ${ }^{\mathrm{PTC}}$ isoform 45 aa of the $\mathrm{C}$-terminal helicase domain are missing, without affecting the ATP binding domain. Transcripts of both isoforms showed increased half-lives in NMD KO cells and reacted strongly to translation inhibition, a hallmark of bona fide NMD targets.

Control of protein synthesis rates is fundamental for maintenance of self-renewal and differentiation ${ }^{37,38}$ and for maintaining an ESC-specific chromatin state ${ }^{39,40}$. ESCs require a downregulation of translation rates and ribosome biogenesis to successfully differentiate ${ }^{40,41}$. We detected a marked increase in translation rates in NMD KO ESCs, which is not solely an effect of increased mRNA abundance due to increased mRNA stability, but directly dependent on increased Eif4a2 levels. This is evident from doubledepletion experiments, in which Eif4a2 depletion in NMD KOs reduces translation and partly rescues differentiation kinetics without restoring NMD function.

Increased protein levels of the Eif4a2 $2^{\text {PTC }}$ isoform and not the full-length protein disrupt the normal translation programme and the exit from naïve pluripotency. This became evident in NMD KO cells in which the PTC exon was excised. In these cells, differentiation kinetics were restored despite increased 
expression of Eif4a2 $2^{\mathrm{FL}}$. Furthermore, only Eif4a $2^{\mathrm{PTC}}$ overexpression elicits clear differentiation delays. The function of Eif4a2 $2^{\mathrm{PTC}}$ appears dependent on the presence of Eif4a2 $2^{\mathrm{FL}}$ because overexpression of Eif4a2 ${ }^{\mathrm{PTC}}$ alone in Eif4a2 KO ESCs has no detectable impact on differentiation. This constitutes evidence that the translation initiation potential of Eif4a2 can be increased by its PTC isoform, which in turn requires the full-length protein to exert its function.

Eif4a2 ${ }^{\text {PTC }}$ protein is detectably expressed in ESCs, but its physiological role remains to be determined. The observed interaction between Eif4a2 $2^{\mathrm{FL}}$ and Eif4a2 ${ }^{\mathrm{PTC}}$ with key pluripotency TFs is of special note. This interaction with the pluripotency circuitry remains to be explored, but could provide a direct link between translation initiation and cell fate control. Such a function accords with recent reports of Sox 2 binding to the translation initiation factor transporter Eif4enif1, and Sox2 binding to RNA in mouse ESCs ${ }^{44,45}$.

NMD is triggered during translation. NMD-mediated regulation of Eif4a2 provides a link back from NMD to translation initiation, thereby establishing a feedback circuit (Fig. 6i). We propose that such a mechanism can be used to balance NMD activation with translational activity. We propose Eif4a2 ${ }^{\text {PTC }}$ to act as a rheostat: Its upregulation upon NMD dysfunction increases translation initiation activity, which in turn directly increases the chances of triggering NMD in successive rounds of Eif4a2-initiated translation. In line with this hypothesis, our data confirm a reported physical interaction between Eif4a2 and Smg factors ${ }^{35}$. Underscoring the importance of the Eif4a2 $2^{\text {PTC }}$ mediated feedback loop to translation, the PTCcontaining exon shows an unexpectedly high level of evolutionary conservation in various mammalian species, including human.

In conclusion, we show that NMD is required for proper restructuring of GRNs during differentiation by targeting multiple transcripts, most prominently the translation initiation factor Eif4a2. Our results place NMD as a central player in shaping transcriptomes to maintain proper cellular identity. We provide evidence for an intricate feedback circuit between NMD and translation. NMD susceptibility is hardwired 
418 in an evolutionarily conserved fashion into the Eif4a2 gene structure and sequence. This direct link

419 between NMD and translation initiation will need to be considered when studying NMD associated

420 phenotypes. Our results are also relevant for improving therapeutic interventions and provide a rational

421 for choosing the appropriate Smg-factor in pharmacological approaches to inhibit NMD activity, 422 depending on the desired strength of NMD inactivation ${ }^{15}$.

\section{AUTHOR CONTRIBUTIONS}

427 EG performed experiments and analyzed data. RS, MR, FTT, LH and MG performed bioinformatic analysis, supervised by $\mathrm{AB}, \mathrm{AvH}$ and $\mathrm{CB}$. MH, JR, SS and LS performed experiments. $\mathrm{CW}$ and $\mathrm{CS}$ analyzed teratoma samples. AC, KFL and AP supported translation measurements. VH and SLA performed half-life measurements. EG, AS and ML designed experiments. ML supervised the study and wrote the manuscript together with EG with feedback from all authors.

\section{ACKNOWLEDGEMENTS}

434 We thank Thomas Sauer and Johanna Stranner at the Max Perutz Labs FACS facility for expert support, Markus Hartl and the Massspec facility for mass-spec analysis and Irmgard Fischer for help with

436 microscopy. NGS was performed at the VBCF. This work was supported by the Austrian Science Fund (FWF;

437 P31334). Martin Leeb is a WWTF VRG group leader (VRG14-006) and was supported by an FWF Schroedinger return fellowship. 


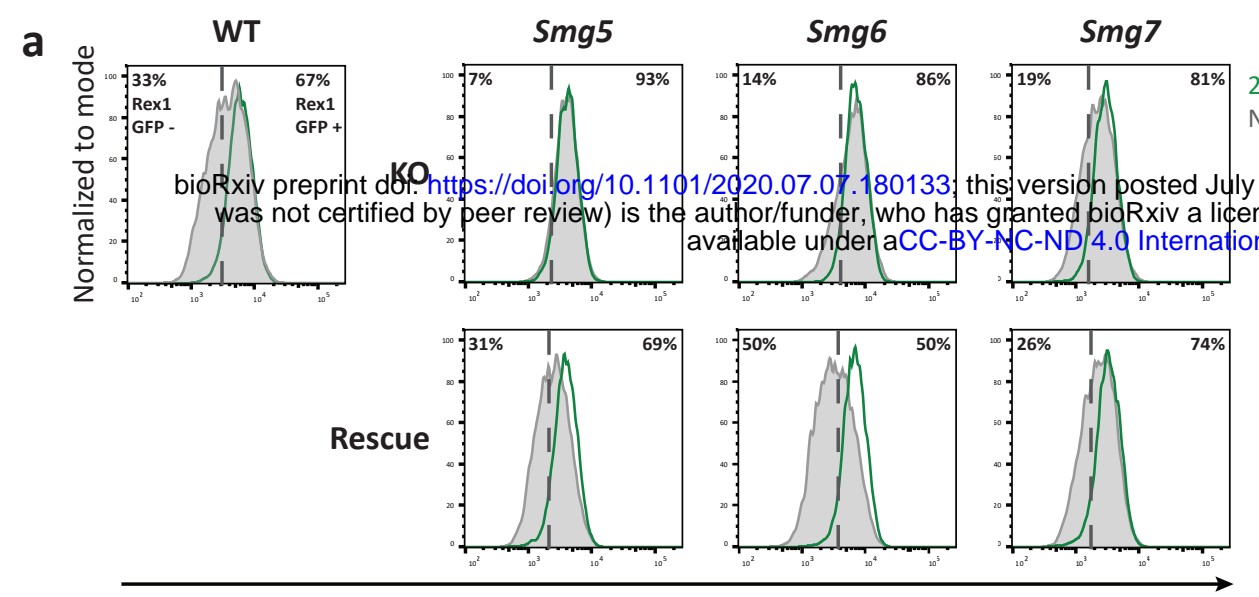

b

Rex1-GFPd2

C
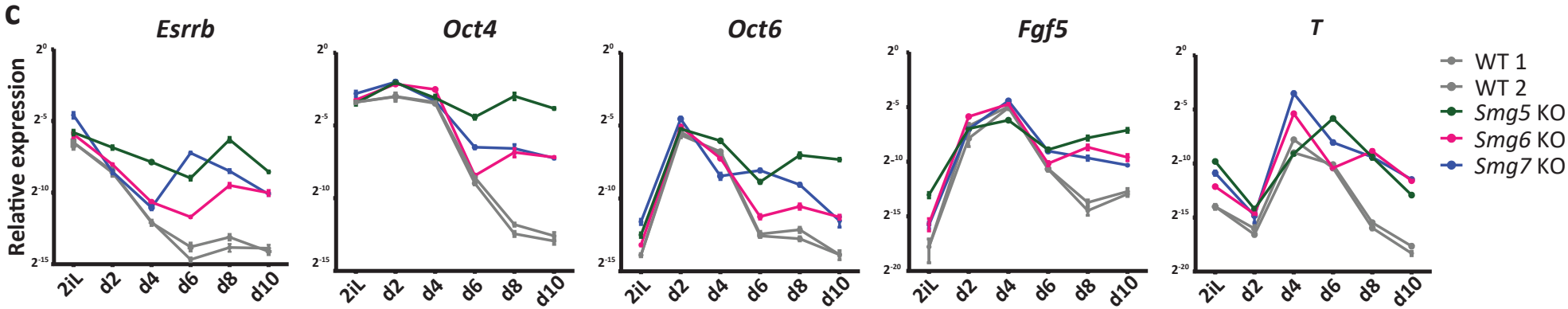

d

e

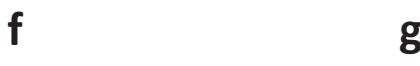

g

$\mathrm{h}$

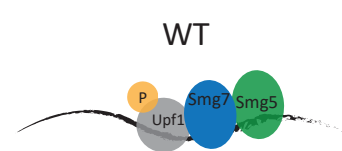

Smg5 KO + siSmg6

Smg6 KO + siSmg7

Smg5 KO + siSmg7

Smg5 KO + siSmg6/siSmg 7

Exonucleolytic cleavage

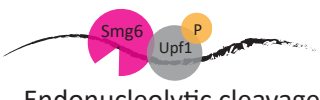

Endonucleolytic cleavage

$\frac{0}{0}$

है
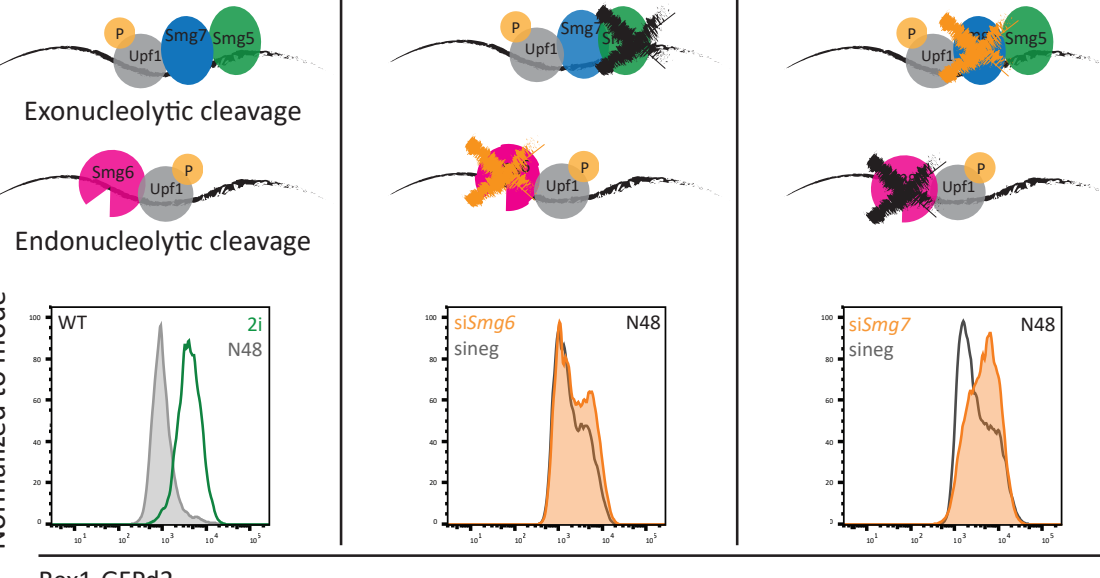

i
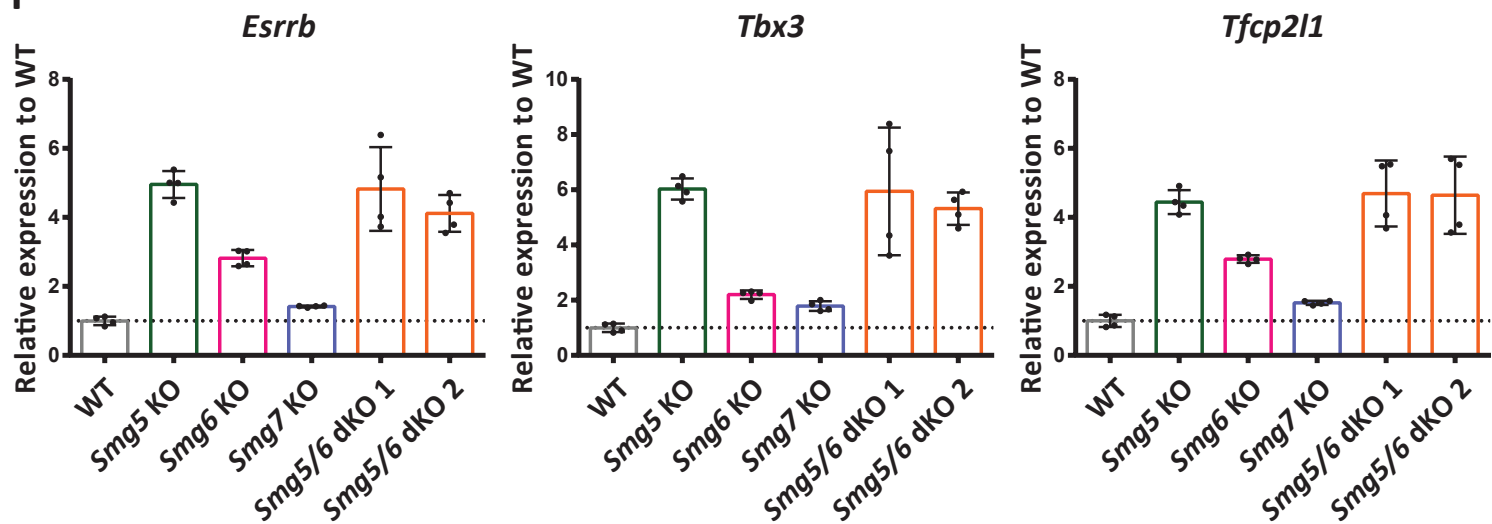

Figure 1 

analysis by FACS $24 \mathrm{~h}$ after induction of differentiation by withdrawing $2 \mathrm{i}$ in N2B27 medium (N24) in NMD KO, NMD rescue and WT ESCs. Green indicates profiles in $2 \mathrm{i}$ and grey indicates N24 profiles. $\mathbf{b}$,

442 Commitment assay in NMD KO and NMD rescue ESCs confirms commitment defects. Cells were re-plated

443 in $2 \mathrm{i}$ medium after $72 \mathrm{~h}$ of differentiation. Only non-committed cells can reinitiate ESC self-renewal.

444 Alkaline phosphatase staining was used to visualize ESC colonies. c, Representative experiment for 445 expression kinetics of the indicated genes during a 10-day embryoid body (EB) differentiation assay. Mean 446 and SD of technical replicates are plotted for each time point. Expression levels were normalized to 60S 447 ribosomal protein L32 Rpl32 (L32). d-h, Rex1-GFPd2 analysis at N48 of WT cells (d) and NMD KO and NMD dKO cells after siRNA KD of Smg6 or Smg7 (e-h). i, Expression of the indicated genes at N24 in NMD KO,

449 Smg5/Smg6 dKO and WT ESCs. Mean and SD are plotted for each cell line, $\mathrm{n}=2$ biological replicates. 450 Expression was normalized first to Actin and results are shown relative to WT. Unpaired t-test was used 451 to calculate $p$-values (**** $p<0.02$ unless indicated in the graphs). 
a

PTC

long 3'UTR

UORF b

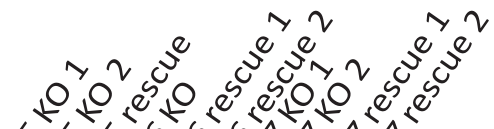

C

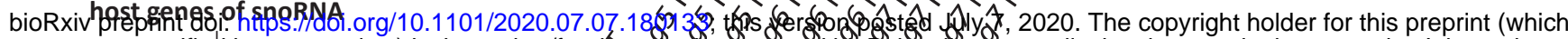

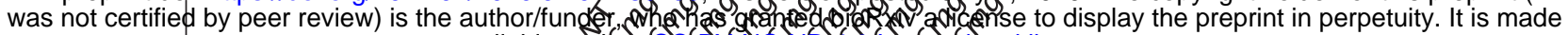

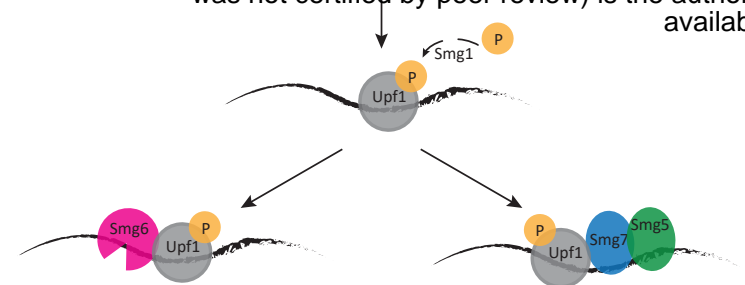

Endonucleolytic cleavage

Exonucleolytic cleavage
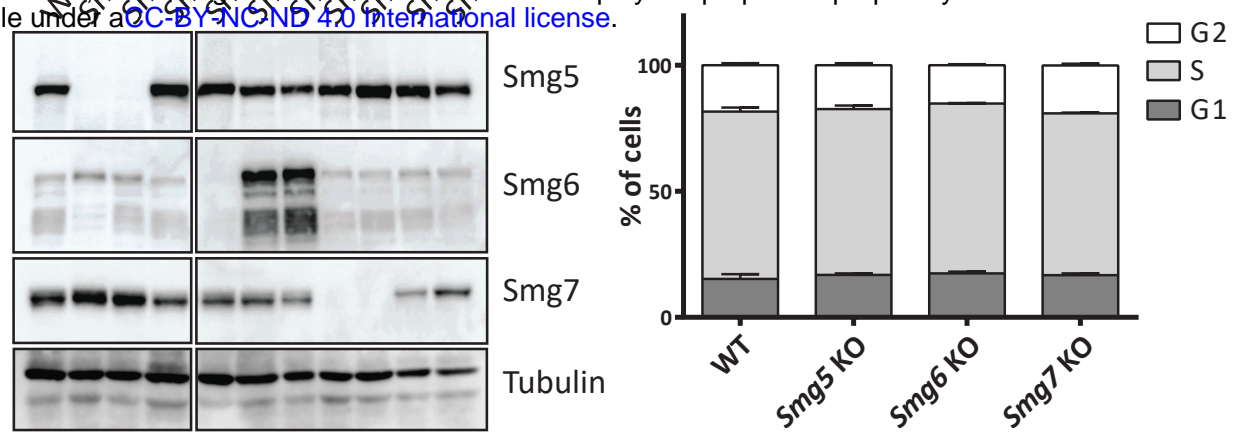

d

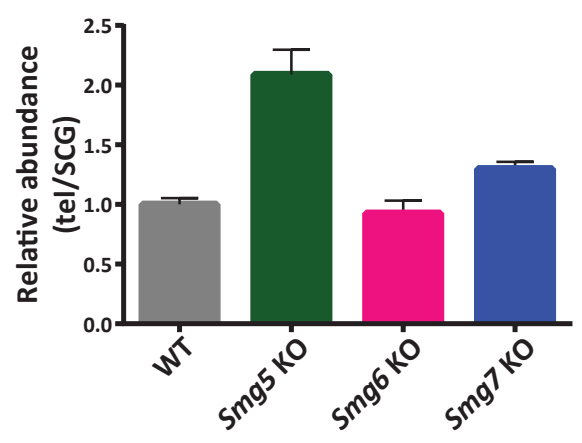

f

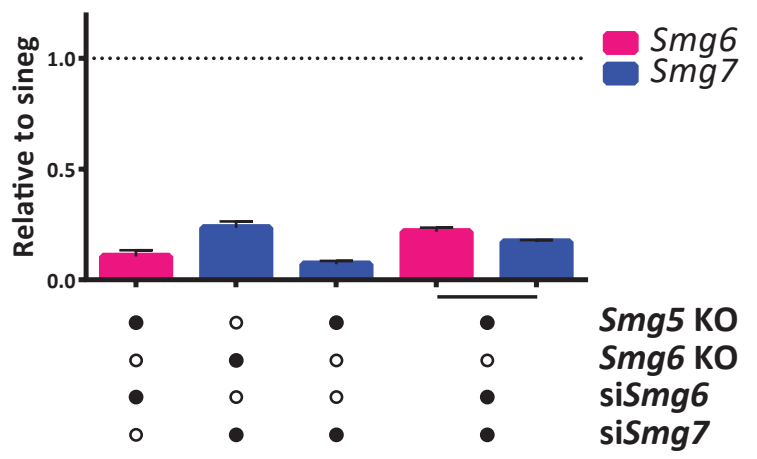

e

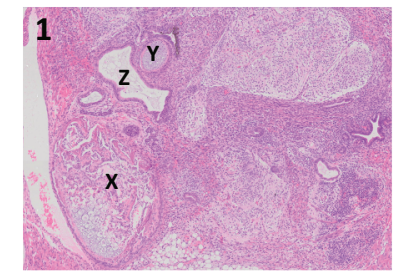

КО

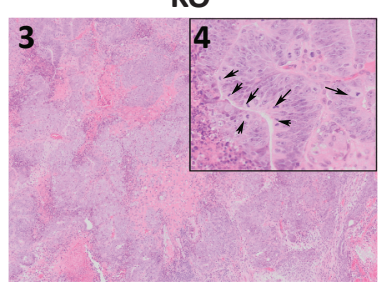

g

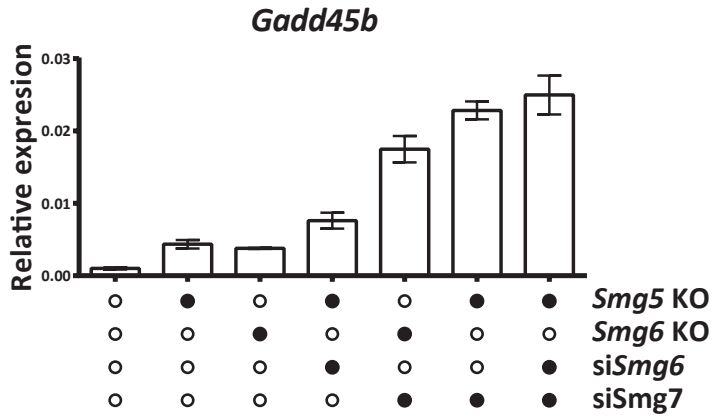


Extended Data Fig. 1 | Hierarchy of defects in exit from naïve pluripotency in NMD-deficient ESCs. a,

454 Illustration of the endo- and exonucleolytic branches of NMD. b, Western blot analysis in WT, NMD KO and NMD rescue ESCs using the indicated antibodies. Tubulin was used as loading control. c, Cell cycle analysis of NMD KO and WT ESCs. Propidium iodide was used to stain the cells and the Watson Pragmatic model was used to calculate the percentage of cells in each cell cycle phase. Mean and SEM are plotted for each cell cycle phase, $n=3$ biological replicates. $\mathbf{d}$, Telomere length calculation in NMD KO and WT ESCs. Mean and SD of technical replicates are plotted for each cell line. Results were normalized to the 36B4 single copy locus. e, Teratomas derived from WT, Smg6 KO and Smg6 rescue ESCs. 1-2: areas of WTderived teratoma showing well differentiated tissues of mesodermal (e.g., $\mathrm{X}$ enchondral ossification; $\mathrm{Y}$ cartilage), endodermal (e.g., Z), and ectodermal (neuronal rosettes; N) origin. 3: Area of Smg6 KO-derived teratomas showing abundant poorly differentiated neuronal tissue; poorly differentiated endodermal and mesodermal tissues are present at lower abundancy (not shown). Abundant mitotic figures present in neuronal tissue (arrows) demonstrate high proliferative capacity (4). 5: Area of Smg6 rescue-derived teratomas showing examples of well differentiated endodermal (Z) and mesodermal (Y: cartilage) tissues. 467 Higher power magnification shows an endodermal duct with different, well developed epithelial cell types 468 (6). $f$, qPCR analysis confirming the siRNA-mediated knockdown of NMD components. Mean and SD of technical replicates are plotted for each cell line. Expression was normalized first to $L 32$. Expression levels

470 after sineg transfection were set to 1. g, Expression levels of Gadd45b in the indicated cells lines. 


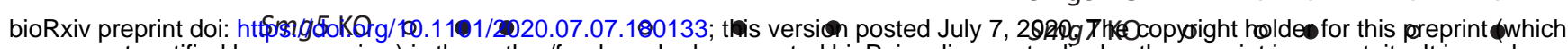
was not certified by Seer 6 (qview) is the author/funder, who has granted bioRxiv a license to display the preprint in perpetuity. It is made
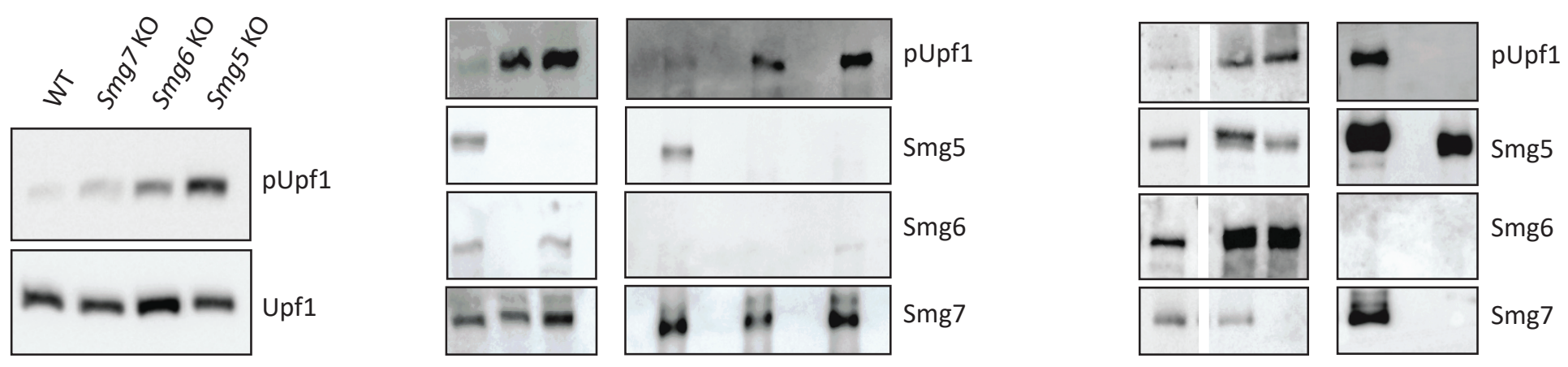

d

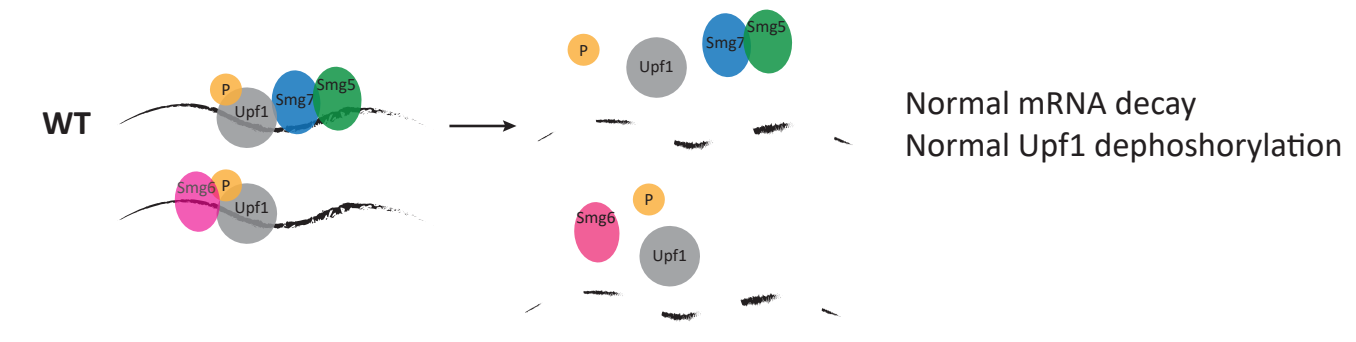

Smg5 ко

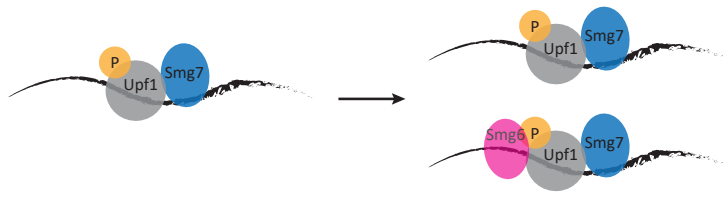

Impaired endo- and exonucleolytic mRNA decay Strong Upf1 dephosporylation impairment \& phenotype

\section{Smg7 ко}

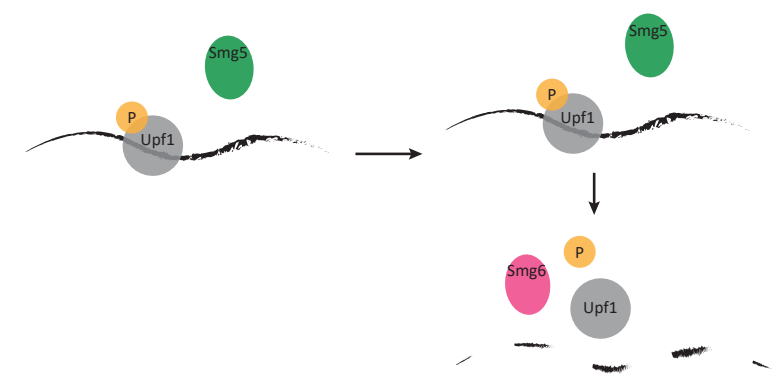

Impaired exonucleolytic mRNA deay pUpf1 bound mRNA can be recognised by Smg6 Mild Upf1 dephosphorylation impairment \& phenotype 
473 Fig. 2 | Smg7 is necessary and sufficient for pUpf1 binding, independently of Smg5. a, Western blot

474 analysis for Upf1 and pUpf1 expression in NMD KO and WT ESCs. b, Western blot showing results of Smg7

475 co-IP in the indicated cell lines after cyanase treatment for RNA removal. Antibodies used are indicated in

476 the figure. c, Western blot analysis of Smg5 co-IP (flag antibody co-IP in Smg5KO ${ }^{\text {Smg5flagrescue }}$ cells) in the

477 indicated cell lines after cyanase treatment. Antibodies used are indicated in the figure. $\mathbf{d}$, Schematic

478 illustration of the proposed interactions between NMD effectors and pUpf1 dephosphorylation in WT,

479 Smg5 and Smg7 KO ESCs that lead to a graded deficiency in NMD and exit from naïve pluripotency. 
a

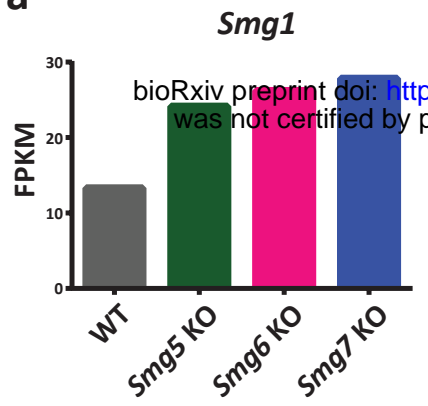

b

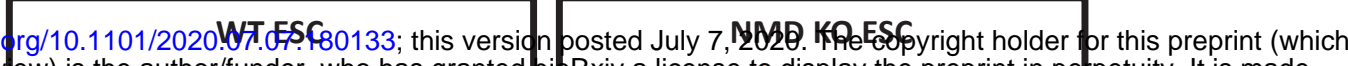

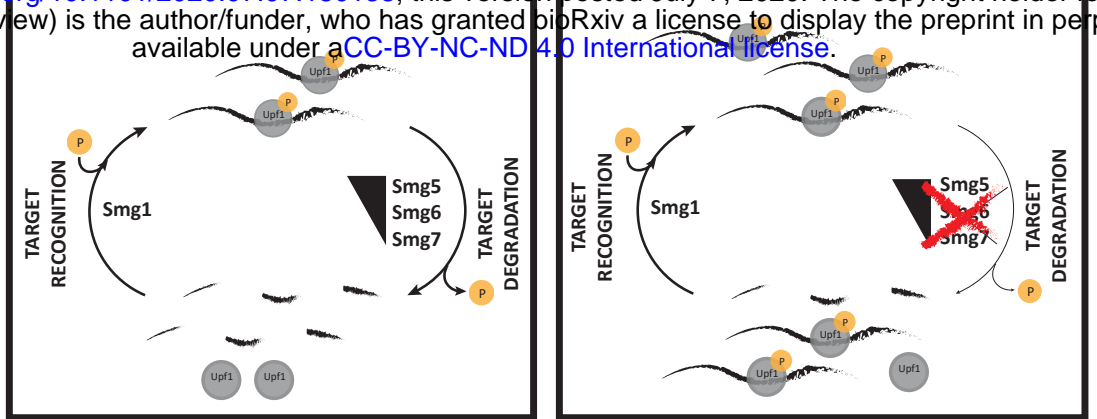

C

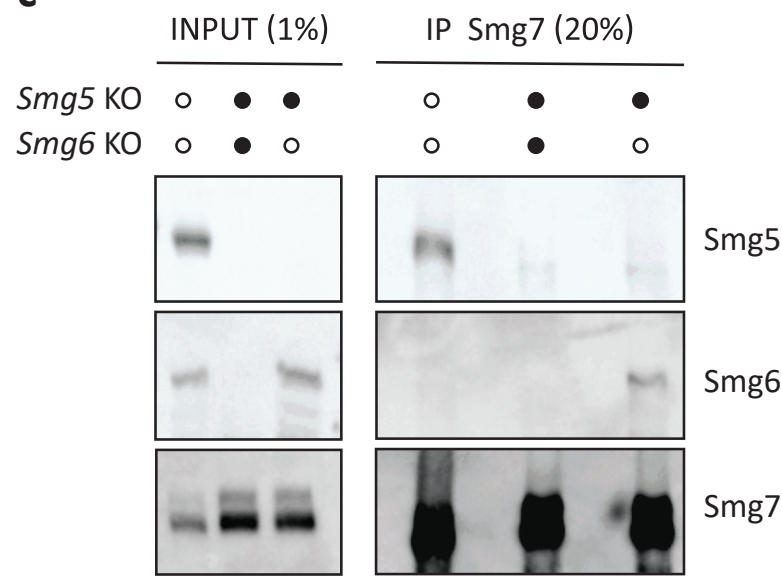

d $\quad$ INPUT (1\%) IP Flag (20\%)

Smg5 KO

Smg KO ० ० • ०

Smg5flag ○ • • •

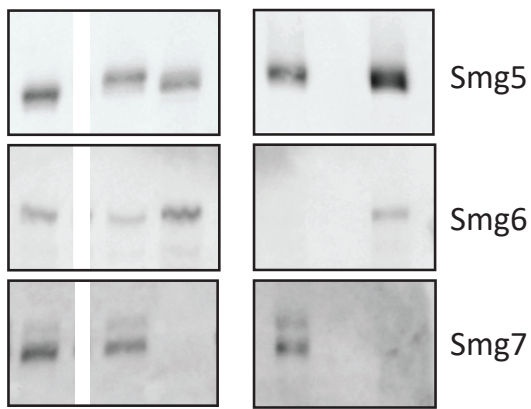


481 Extended Data Fig. 2| Smg7 is necessary and sufficient for pUpf1 binding, independently of Smg5. a,

482 Expression levels (FPKM) of Smg1 derived from RNA-seq in WT and NMD KO ESCs in 2i. Mean values

483 between biological duplicates are plotted. b, Schematic representation of phosphorylation and

484 dephosphorylation cycle of Upf1 in WT and NMD KO ESCs. c, Western blot of Smg7 co-IP in the indicated

485 cell lines without cyanase treatment. Antibodies used are indicated in the figure. $\mathbf{d}$, Western blot analysis

486 of Smg5 co-IP (flag antibody) in the indicated cell lines without cyanase treatment. Antibodies used are

487 indicated in the figure. $\mathbf{c}$ and $\mathbf{d}$ are parallel experiments to those shown in Figure $2 \mathrm{~b}$ and $\mathrm{c}$. 
a

b
C

$$
\mathrm{N} 24(\mathrm{p} \leq 0.01)
$$

$2 \mathrm{i}(\mathrm{p} \leq 0.01)$

作 wis not certified by peer review) is the author/funder, who has granted'bionxiv a license to display the preprint in perpetuity. It is made
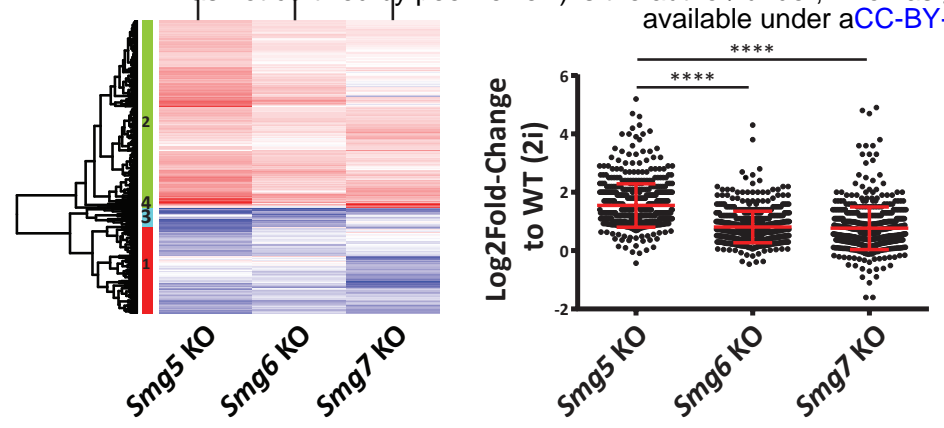

e

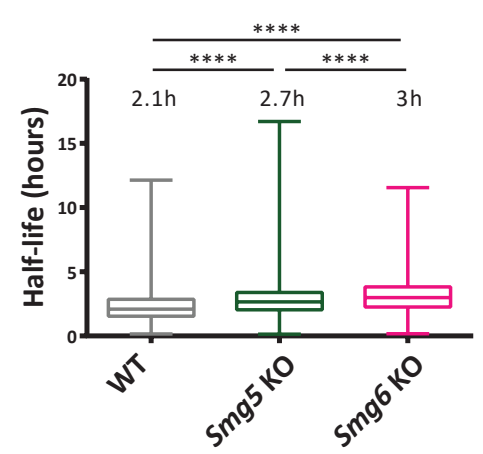

f

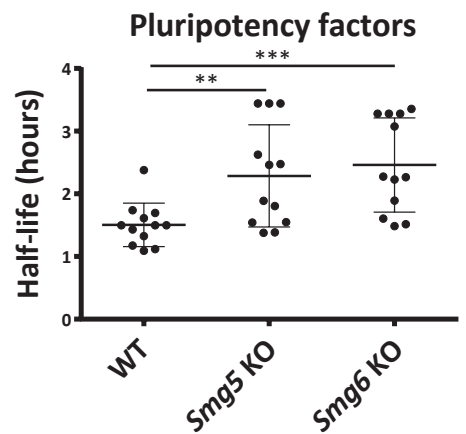

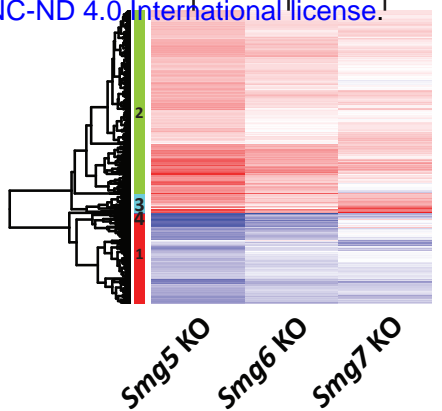

g

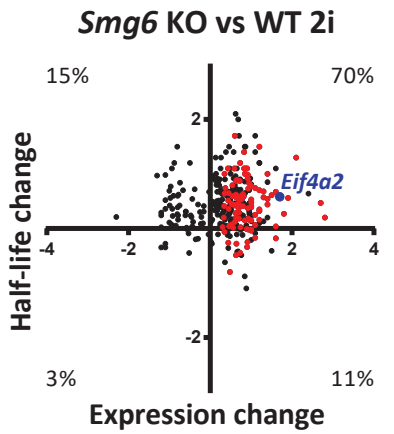

- Transcripts from Figure 3a

- Upreregulation:

Smg5 KO >Smg 6 KO >Smg7 KO

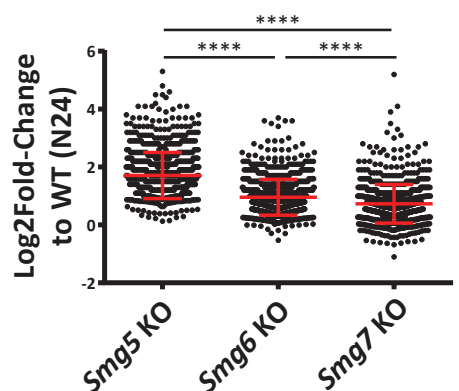

h

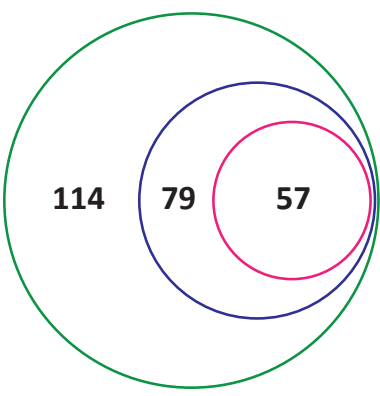

250 transcripts upregulated in all NMD KO ESCS

136 transcripts upregulated with increased half-life in Smg5 and Smg6 ESCs

57 transcripts showing increased expression according to graded phenotypes 


\section{Fig. 3 | Integrating transcriptome-wide gene expression with mRNA half-life analyses identifies relevant} NMD targets during the exit from naïve pluripotency. a, Differentially expressed genes between NMD KO and WT ESCs in self-renewal (2i) (p-adj. $\leq 0.01)$. $p$-adj. values were calculated testing against the null hypothesis of a fold change $<|1.5|$. b, Log2fold change of deregulated transcripts in $2 \mathrm{i}$ belonging to clusters 2 and 4 (564 transcripts). Mean and SD are indicated in the graph. Unpaired t-test was used to calculate p-values. $(* * * * p$-value $<0.0001)$. c, Differentially expressed genes between NMD KO and WT ESCs at N24 (p-adj. $\leq 0.01$ ). p-adj. values were calculated testing against the null hypothesis of a fold change $<|1.5|$. d, Log2fold change of deregulated transcripts at N24 belonging to clusters 2 and 3 (808 transcripts). Mean and SD are indicated in the graph. Unpaired t-test was used to calculate $\mathrm{p}$-values. (**** p-value < 0.0001). e, Half-life analysis in WT, Smg5 KO and Smg6 KO ESCs. Half-life was calculated in hours based on a pulse chase time course. Mean and SD are indicated in the graph. Paired t-test was used to calculate p-values $(* * * *$ p-value $<0.0001)$. f, Half-life of pluripotency factors (Esrrb, Klf2, Klf5, Nanog, Pou5f1, Sall4, Sox2, Stat3, Tbx3) in WT, Smg5 KO and Smg6 KO ESCs. Half-lives were calculated in hours. Mean and SD are indicated in the graph. Paired t-test was used to calculate $p$-values $\left({ }^{* *} p\right.$-value $=0.006$, *** $p$-value $=0.0006) \cdot \mathbf{g}$, Transcriptome-wide comparison of changes in mRNA expression and half-life in Smg6 KO. Half-life changes between WT and Smg6 KO for the 321 out of 516 transcripts upregulated in NMD KO ESCs for which half-lives could be calculated were calculated (see Figure 3a). 150 of these 321 transcripts showed an increase in half-life $(\log 2 \mathrm{FC} \geq 0.2)$ in both Smg5 and Smg6 KOs. Transcripts behaving in accordance with the graded phenotype (Smg5 > Smg6 > Smg7) are depicted in red. $\mathbf{h}$, Schematic illustration of the approach used to identify NMD targets relevant for the differentiation phenotype in ESCs. First significantly upregulated transcripts (see Figure 3a) with measurable half-lives in WT and Smg KOs were selected (250). 136 transcripts showed a concomitant increase in half-life in Smg5 and Smg6 KOs ( $\log 2 \mathrm{FC} \geq 0.2)$. 57 of these transcripts were upregulated according to the graded phenotypes. 


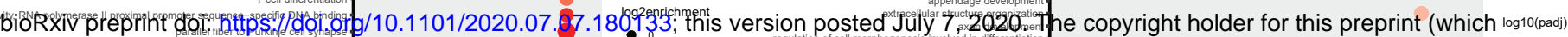

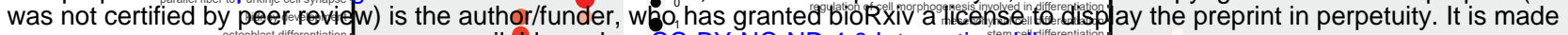

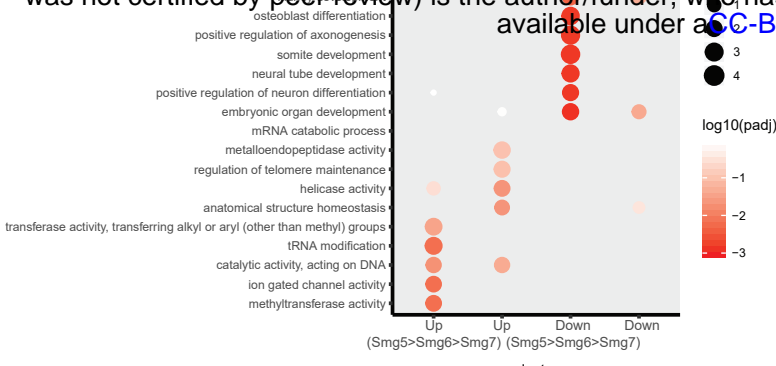

cluster

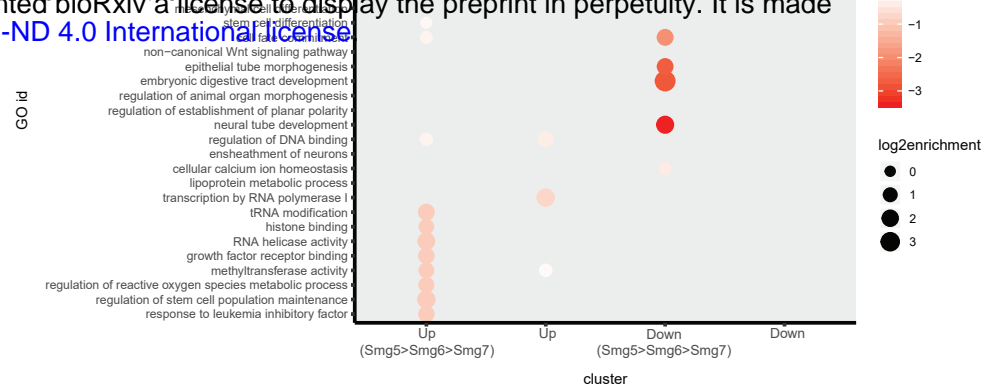

C

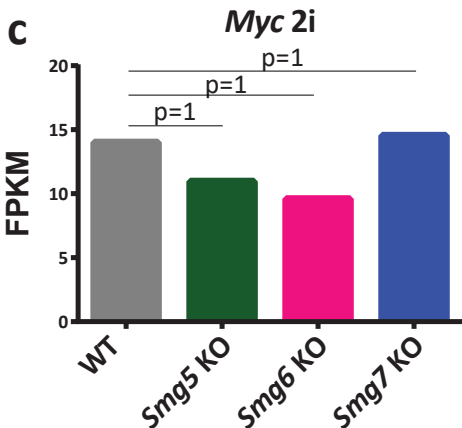

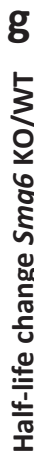

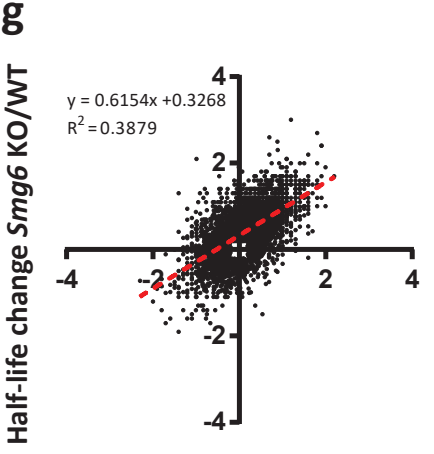

Half-life change Smg5 KO/WT d

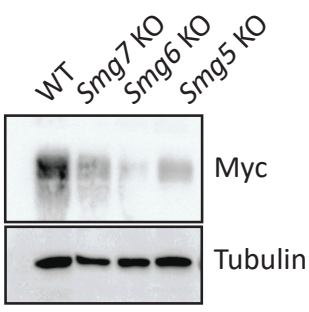

e

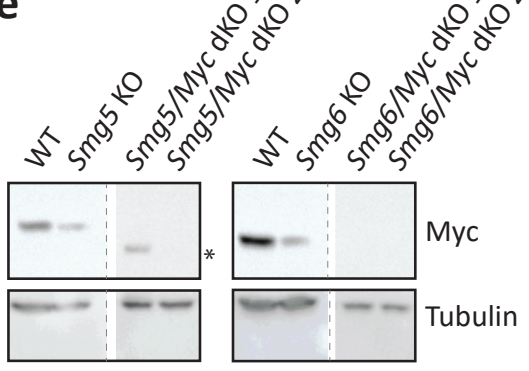

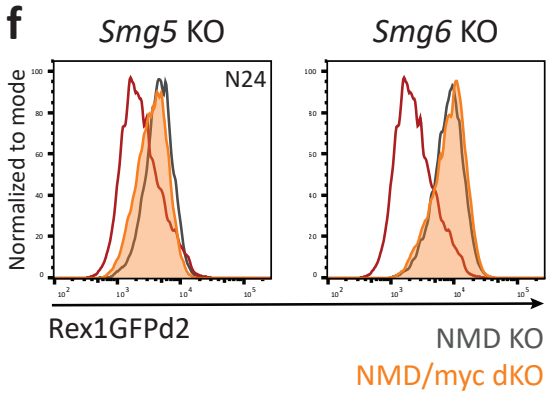

WT h

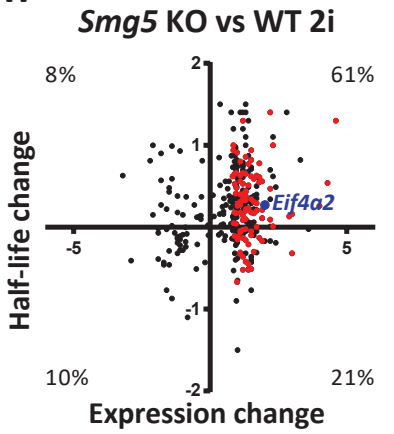

- Transcripts from Figure 3a

Upreregulated transcripts in

Smg5 KO >Smg6 KO>Smg7 KO

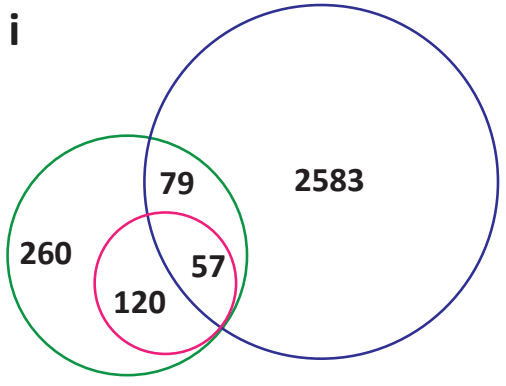

516 transcripts upregulated in all NMD KO ESCs

2719 transcripts with increased half-life in Smg5 and Smg6 ESCs

177 transcripts showing increased expression according to graded phenotypes 
Extended Data Fig. 3| Integrating transcriptome-wide gene expression with mRNA half-life analyses identifies relevant NMD targets during the exit from naïve pluripotency. a, Significantly enriched GO terms of differentially expressed transcripts in NMD KOs in 2i. b, Similar to (a) for differentially expressed transcripts at N24. c, Expression levels of c-myc in NMD KO and WT ESCs in RNA-seq. Mean values between biological duplicates are plotted. $\mathbf{p}$-adj. values are indicated above each bar. $\mathbf{d}$, Western blot analysis in NMD KO and WT ESCs using the antibodies indicated. Tubulin was used as loading control. e, Western blot analysis confirming absence of myc protein in NMD/myc dKO ESCs. Antibodies are indicated in the figure. Tubulin was used as loading control. * band corresponds to truncated protein. f, Rex1-GFPd2 FACS profile at N24 in NMD KO and NMD/myc dKO cells. FACS profile of NMD KO cells is grey, orange profile shows NMD/myc dKO cells and red profile shows WT. $g$, Global analysis of NMD-induced half-life change in Smg5 KO and Smg6 KO ESCs (all transcripts with a half-life change $\geq|0.2|$ in Smg5 or Smg6 KO ESCs are plotted. Half-life change was calculated for 7,451 transcripts. Slope and R2 values are shown in the figure. $\mathbf{h}$, Transcriptome-wide analysis of changes in mRNA expression and half-lives in Smg5 KO. Half-life changes could be calculated for 321 out of the 516 transcripts upregulated in NMD KO ESCs (Figure 3a). 182 of the 516 transcripts showed a concomitant increase in half-life (half-life change $\geq 0.2$ ). Transcripts behaving according to the graded phenotype are depicted in red. i, Venn diagram showing 516 transcripts upregulated in all NMD KO ESCs intersected with 177 transcripts showing graded increase in expression in NMD factor KOs (Smg5 > Smg6 > Smg7) and 2,719 transcripts with increased half-lives in both Smg5 and Smg6 KO ESCs. 
bioRxiv preprint doi: https://doi.org/10.1101/2020.07.07.180133; this version posted July 7, 2020. The copyright holder for this preprint (which was not \&ertified by peer review) is the author/funder, who has granted bioRxiv a license to display the preprint in perpetuity. It is made

Smg6 KO $2 \mathrm{i}$

WT $2 \mathrm{i}$

$$
0-2100
$$

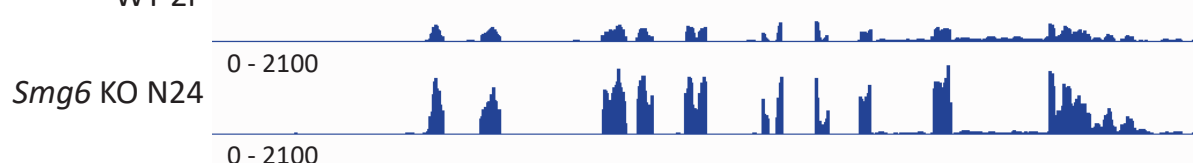

WT N24

$$
0-2100
$$

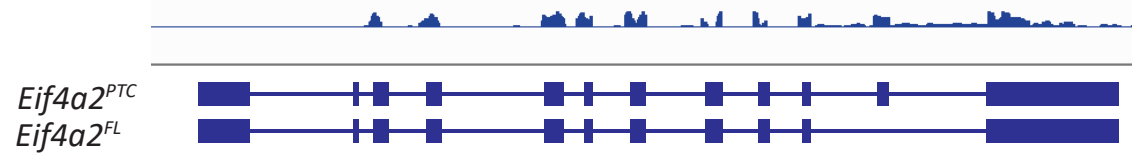

C
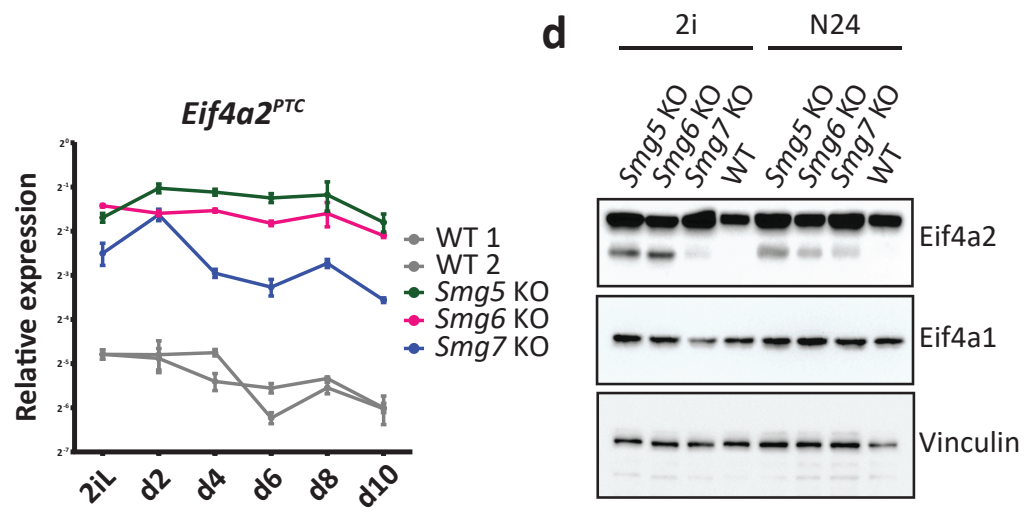

Eif4a2 gene structure

Mouse

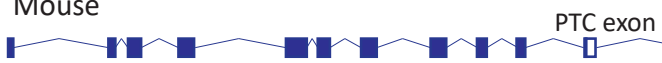

Human

PTC exon

Dog

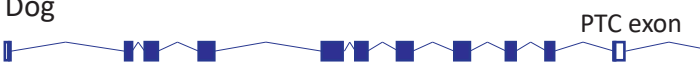

f

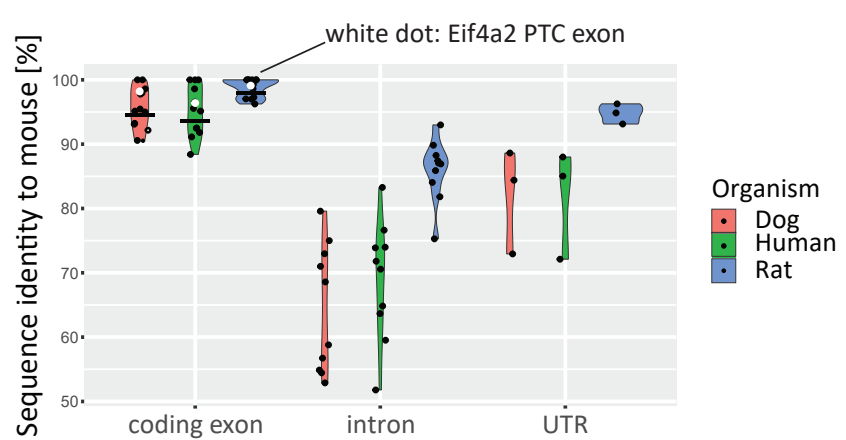

g

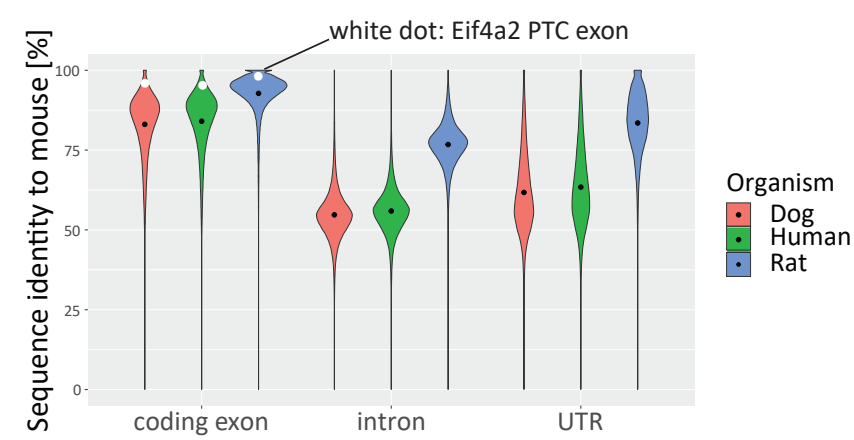


534 Fig. 4 | Eif4a2 is a bona-fide NMD target in ESCs. a, Genome browser view of the Eif4a2 locus visualizing

535 RNA-seq results in Smg6 KO and WT ESCs in 2i and at N24. * indicates qPCR primers amplifying all Eif4a2

536 isoforms. \# indicates qPCR primers amplifying the Eif4a2 ${ }^{P T C}$ isoform. b, Expression levels of the indicated

537 genes after treatment with CHX or DMSO in WT ESCs. Mean and SD are plotted, $\mathrm{n}=4$ biological replicates.

538 Unpaired t-test was used to calculate $\mathrm{p}$-values $(* * * * \mathrm{p}<0.0001)$. $\mathrm{c}$, Representative experiment for

539 expression kinetics of the indicated genes during an EB differentiation assay as in Figure 1c. Mean and SD

540 from technical replicates are plotted for each time point. Expression levels were normalized to L32. d,

541 Western blot analysis of Eif4a2 and Eif4a1 protein levels in NMD KO and WT ESCs in self-renewal (2i) and

542 at N24. Vinculin was used as loading control. e, Schematic representation of the gene structure of Eif4a2

543 in mouse, human and dog. $\mathbf{f}$, Percentage of sequence identity to mouse of Eif4a2 between dog, rat and

544 human. White dots indicate the PTC-containing exon of Eif4a2 and black lines indicate the mean. $\mathrm{g}$,

545 Percentage of sequence identity to mouse of the transcriptome between dog, rat and human. White

546 asterisks indicate the PTC-containing exon of Eif4a2 and the black dots indicate the mean. 
a

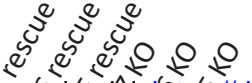

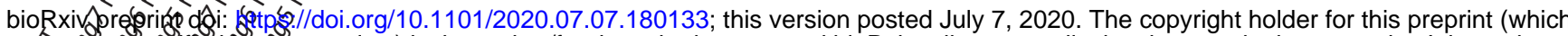

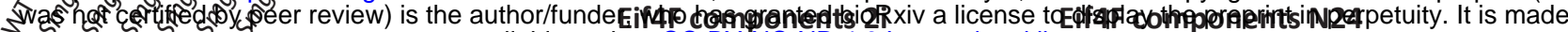

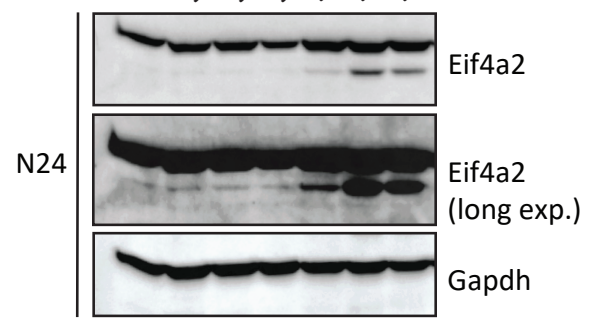

d

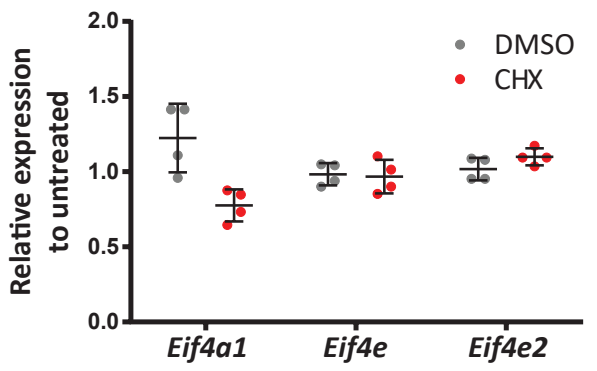

f

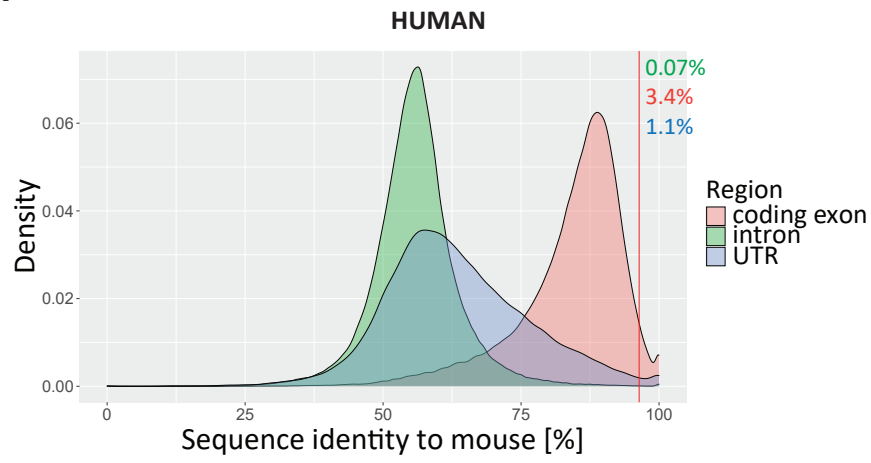

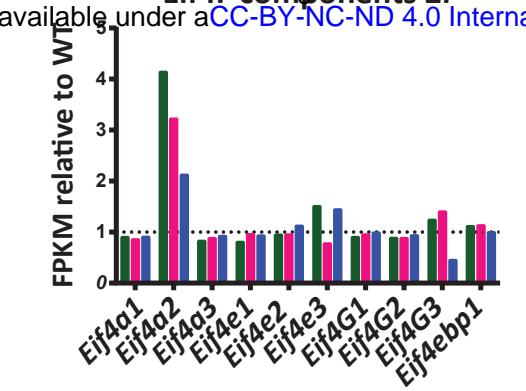

e

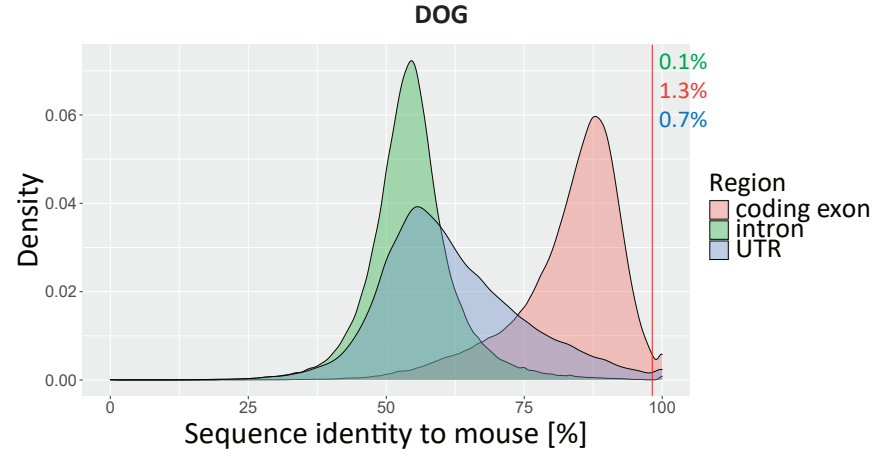

g

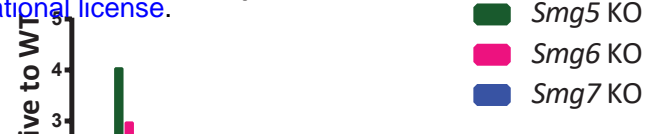

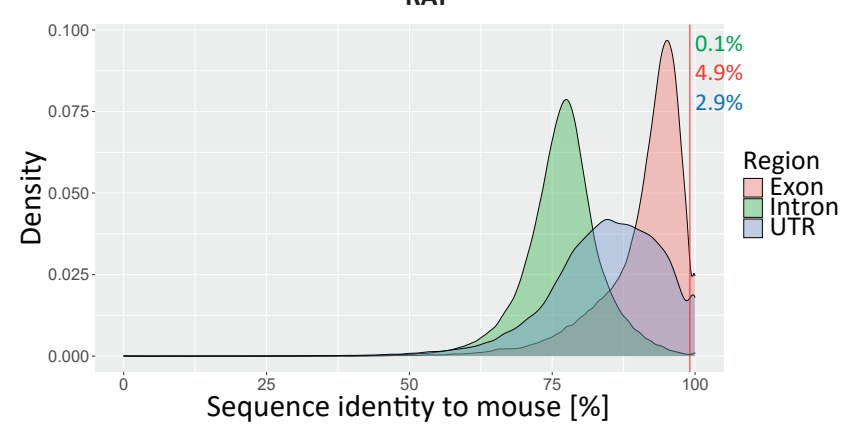


548 Extended Data Fig. 4 | Eif4a2 is a bona-fide NMD target in ESCs. a, Western blot analysis for Eif4a2

549 protein levels in NMD KO, NMD rescue and WT cells at N24. Gapdh was used as loading control. b, 550 Expression of Eif4F complex components and regulators in NMD KO ESCs relative to WT ESCs in self-

551 renewal (2i). FPKM values from RNA-seq analysis are shown, see Figure 3a. c, Expression of Eif4F complex

552 components and regulators in NMD KO cells relative to WT cells at N24. Values from RNA-seq analysis,

553 see Figure 3c. d, Expression levels of the indicated genes after treatment with CHX or DMSO in WT ESCs,

554 assayed by RT-qPCR. Mean and SD are plotted, $n=2$ biological replicates. e-g, Densities of the distributions

555 of the sequence identities of indicated regions of the mouse transcriptome compared to dog (e), human

556 (f) and rat (g). Red line indicates identity of PTC-containing Eif4a2 exon. Percentage of genomic sequences

557 with higher conservation than PTC-containing exon of Eif4a2 are indicated in the graphs. 


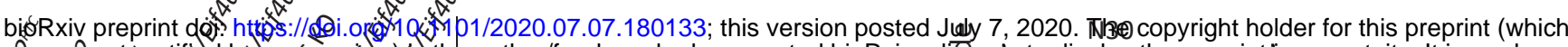

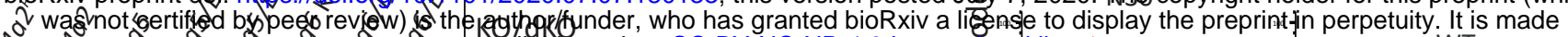

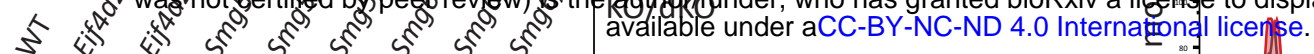
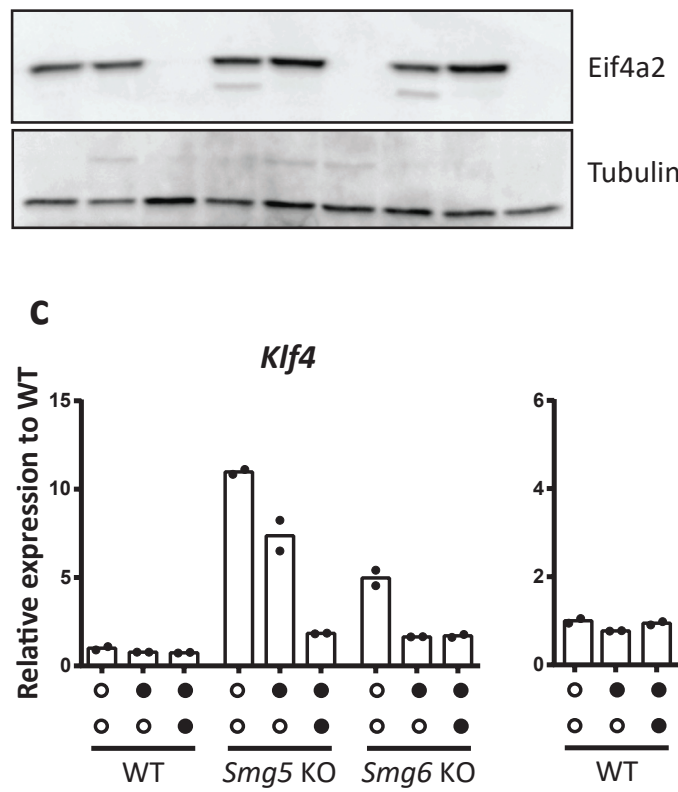

d

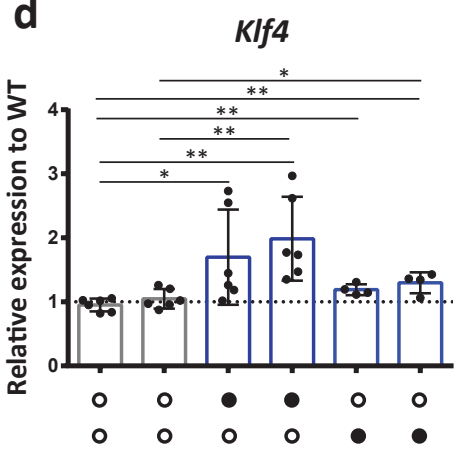

e

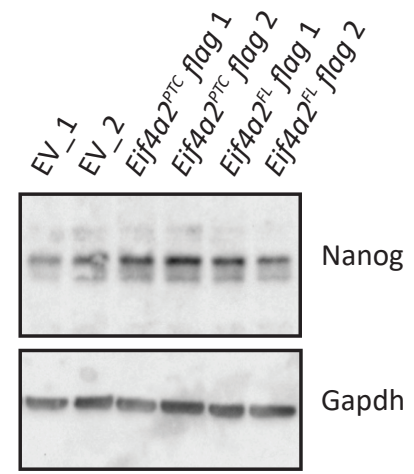

f
Nanog

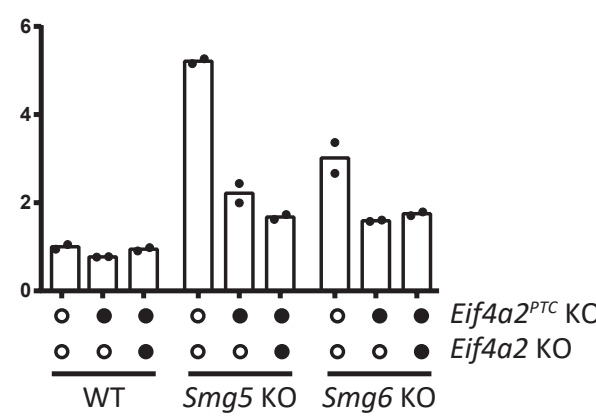

Eif4a2 ${ }^{\text {PTC }} \mathrm{KO}$

Eif4a $2 \mathrm{KO}$

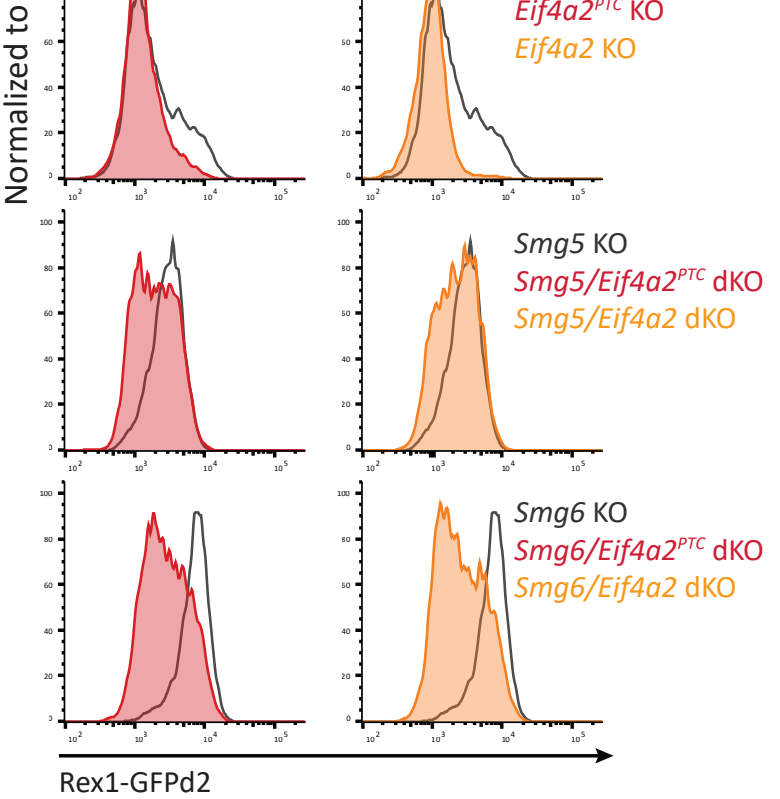

Tfcp2/1

Nanog
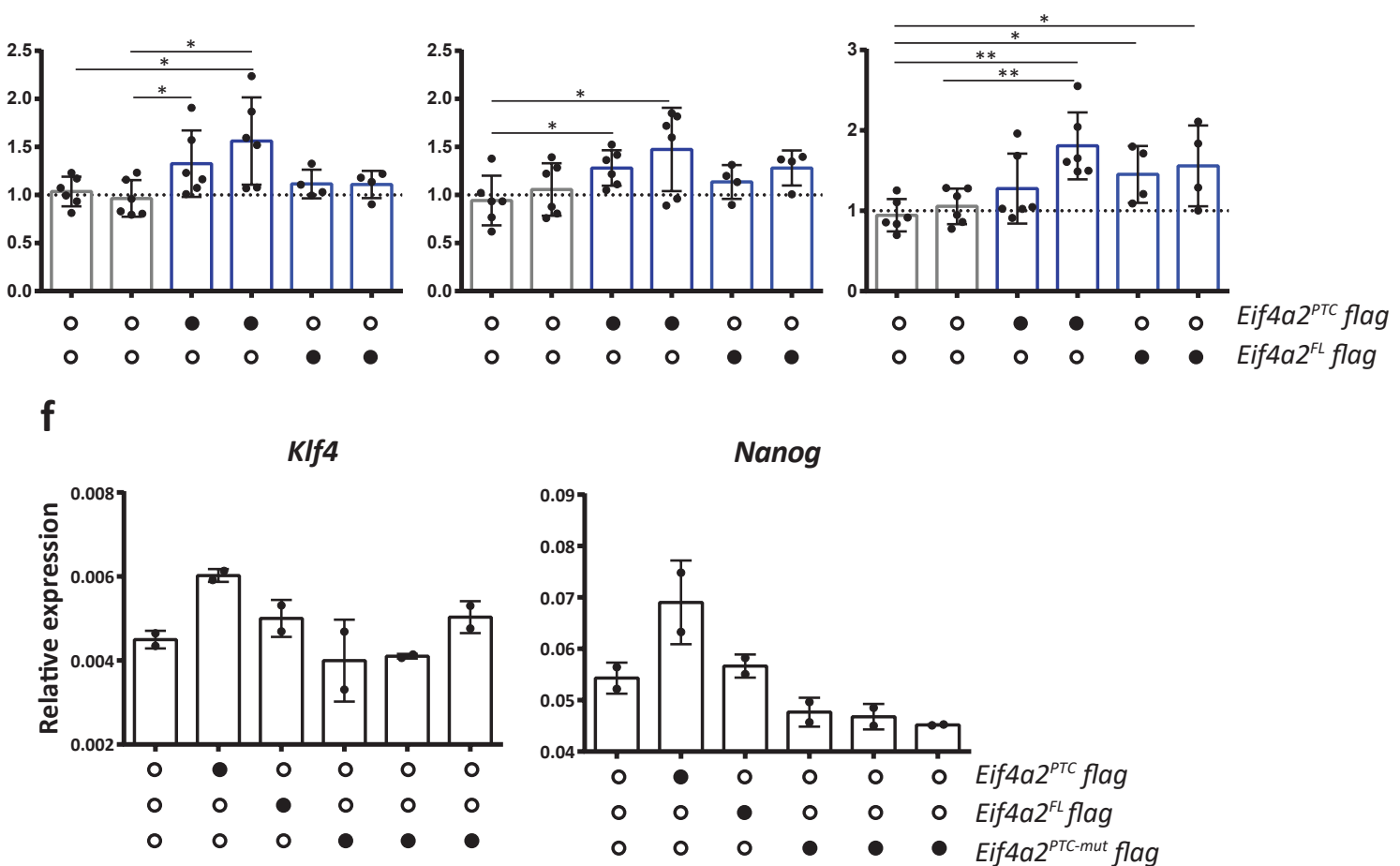

analysis at N30 in NMD KO and NMD/Eif4a2 dKO ESCs. Grey indicates FACS-profiles of WT cells, red

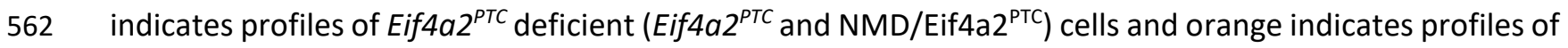

563 Eif4a2 deficient (Eif4a2 KO and NMD/Eif4a2 dKO) cells. c, Representative experiment for the expression

564 of indicated genes at N30 in NMD KO and NMD/Eif4a2 dKO cells. Mean of technical replicates plotted for

565 each cell line. Expression was normalized first to $L 32$ and then to WT cells. d, Expression of the indicated

566 genes at N30 in Eif4a2 overexpressing and WT cells. Mean and SD are plotted for each cell line, n=3

567 biological replicates. Expression was normalized first to housekeeping genes (actin or $L 32$ ). The expression

568 level in WT cells was set to 1 . Unpaired t-test was used to calculate $p$-values $(* p<0.05, * * p<0.005)$. e,

569 Western blot analysis for Nanog in Eif4a2 overexpressing and WT cells at N24. Gapdh was used as loading of technical replicates are plotted for each cell line. 


\section{a}

PTC

g2 g3

d

biokxiv preprint dol: hittps://dolo.org//10.1 was not certifififf(\$)

b

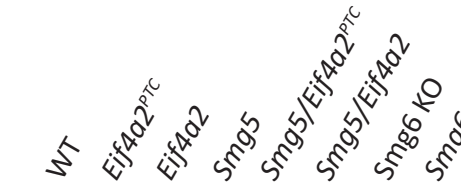

is available under aCC-BY-NC-ND 4.0 International licenge.e.

$$
\text { C }
$$

$\mathrm{KO} / \mathrm{dKO}$

N24

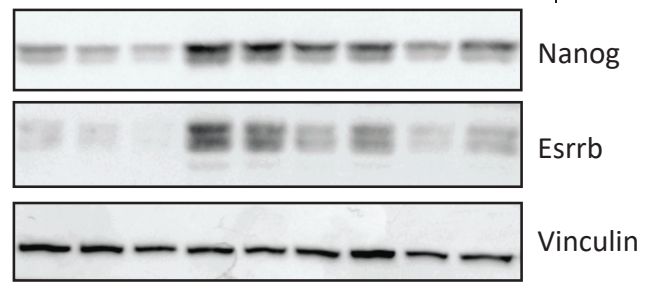

e
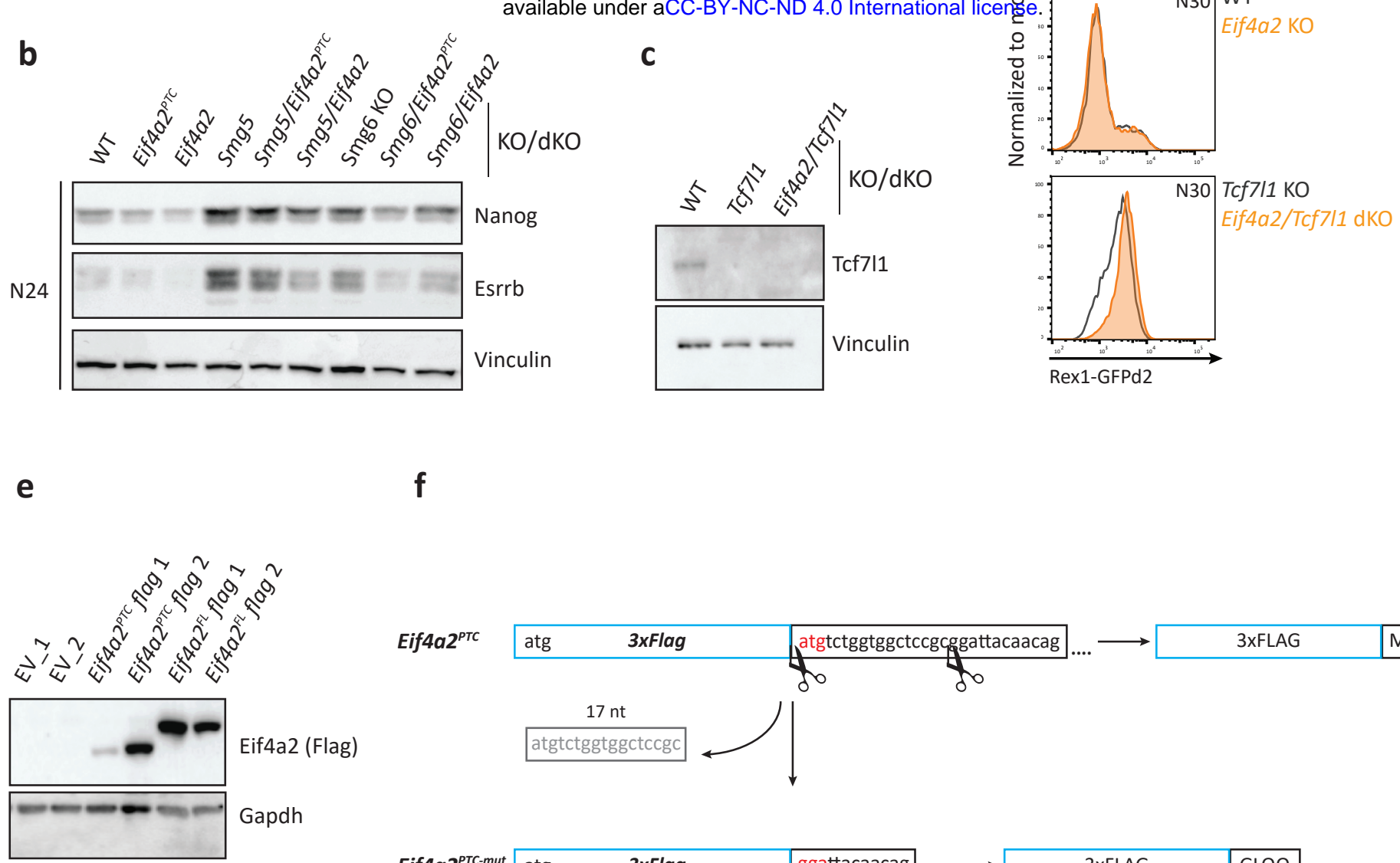

Eif4a2 ${ }^{\text {PTC }}$
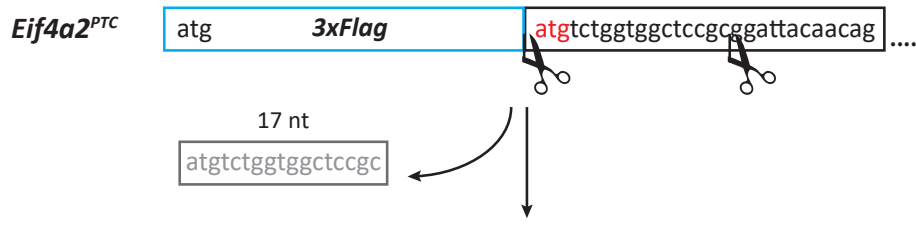

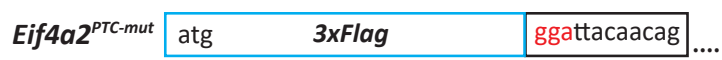

3xFLAG $\ldots \longrightarrow$

GLQQ

g

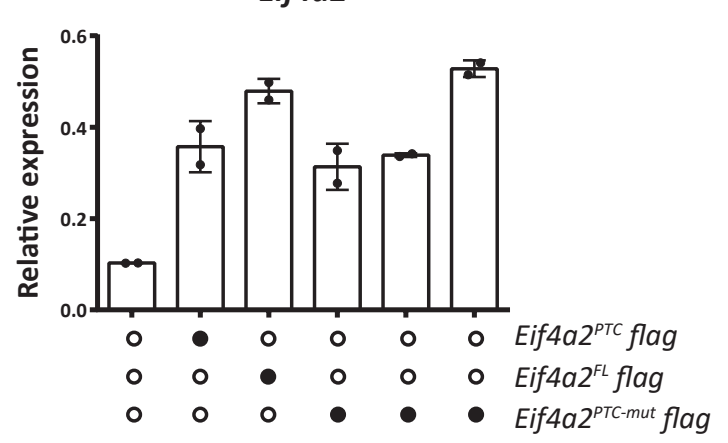


573 Extended Data Fig. 5| Eif4a2 is causative for defects in exit from naïve pluripotency in NMD-deficient

574 ESCs. a, Schematic view of the Eif4a2 gene. gRNAs (g1 to g3) used to generate Eif4a2 and Eif4a2 ${ }^{\text {PTC }}$

575 depletions are indicated. g1 and g3 were used together to delete all Eif4a2 isoforms. g2 and g3 were used

576 together to delete the Eif4a2 ${ }^{\text {PTC }}$ isoform. b, Western blot analysis at N24 in the annotated cell lines for

577 pluripotency markers Esrrb and Nanog. Vinculin was used as loading control. c, Western blot analysis for

578 Tcf7l1 expression in the indicated cell lines. Vinculin was used as loading control. d, Rex1-GFPd2 analysis

579 at N30 in WT (grey), Eif4a2 KO (orange), Tcf7l1 KO (grey) and Eif4a2/Tcf7l1 dKO (orange) cells. e, Western

580 blot analysis of Eif4a2 (flag antibody) in Eif4a2 overexpressing Eif4a2 KO cells. EV transfection shows no

581 signal. Gapdh was used as loading control. f, Schematic illustration of the mutation introduced into the

582 Eif4a2 ${ }^{\text {PTC }}$ isoform. g, Expression of Eif4a2 at N16 in Eif4a2 overexpressing pools and WT cells. Mean and

583 SD of technical replicates are plotted for each cell line. 


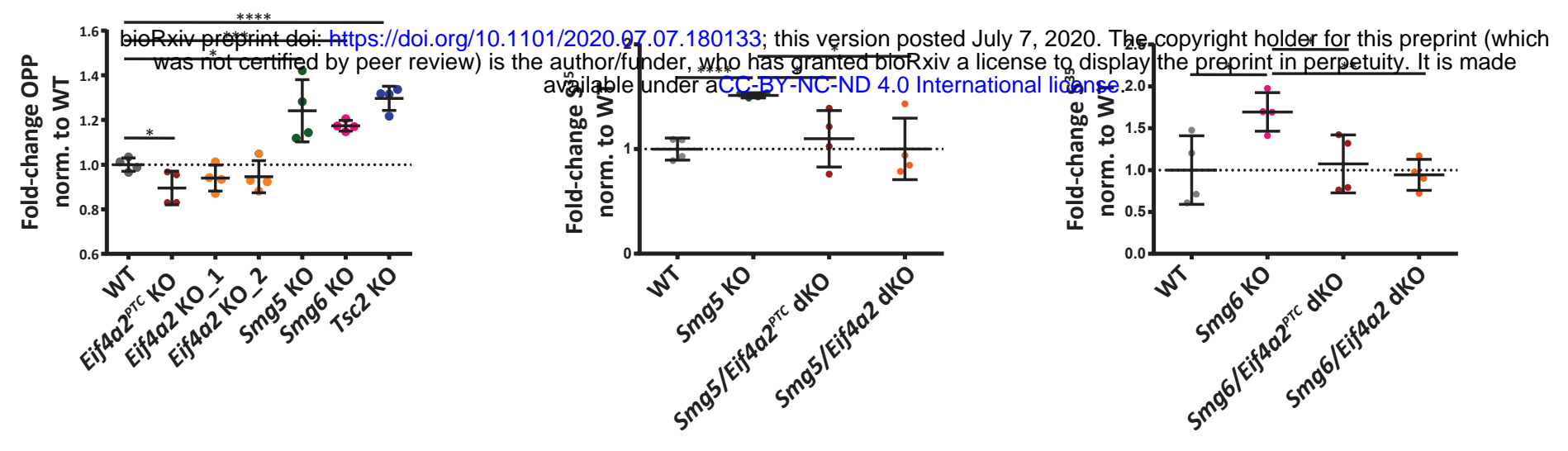

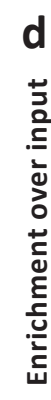
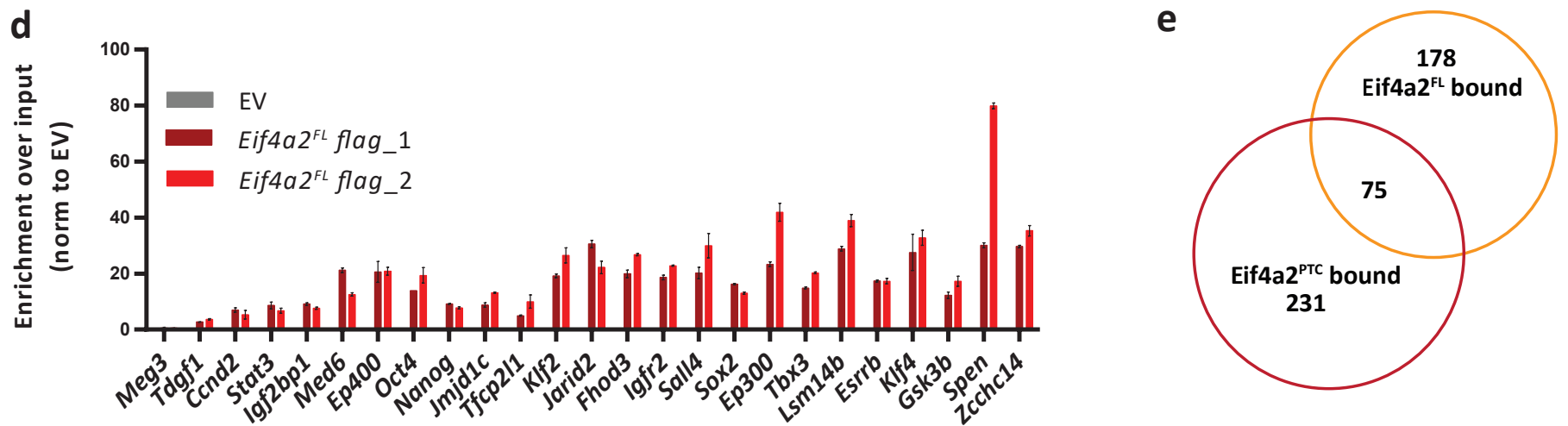

f

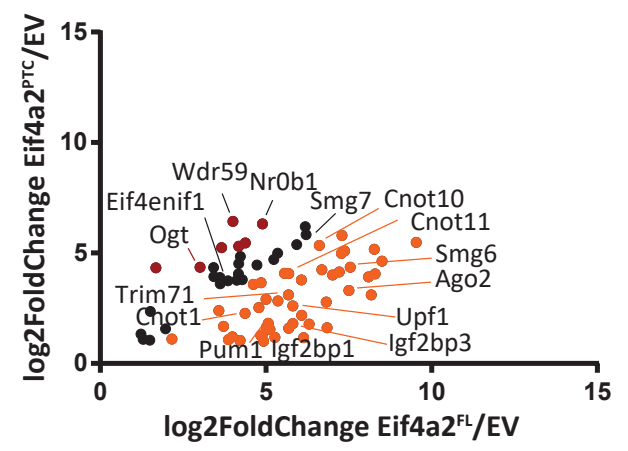

- Bound by Eif4a2 $2^{\mathrm{FL}}>$ Eif4a2 ${ }^{\text {PTC }}$

- Bound by Eif4a2 $2^{\mathrm{PTC}}>$ Eif4a2 $2^{\mathrm{FL}}$

- Bound by Eif4a2 $^{\mathrm{FL}}=$ Eif4a2 $2^{\mathrm{PTC}}$ g

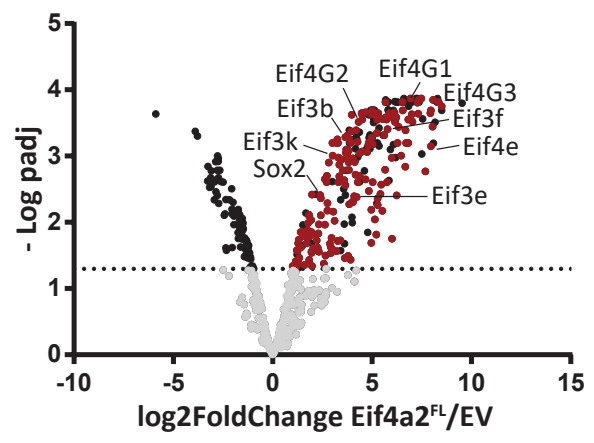

- Eif4a $2^{\mathrm{FL}}$ specific h

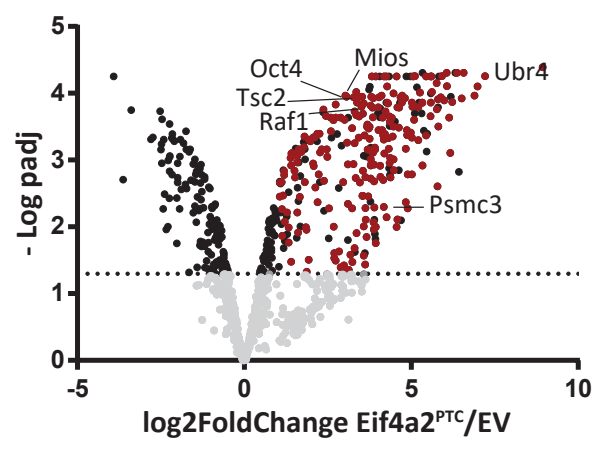

- Eif4a2 ${ }^{\text {PTC }}$ specific

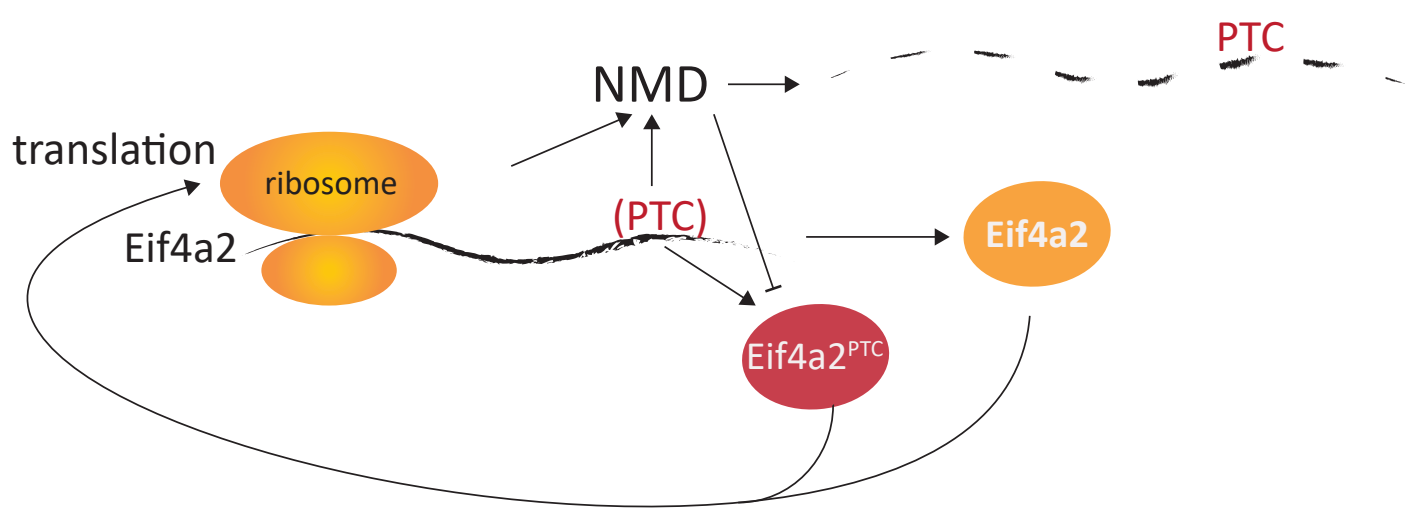



plotted, $\mathrm{n}=4$ biological replicates. Unpaired t-test was used to calculate $\mathrm{p}$-values $\left(* * * * \mathrm{p}<0.0001,{ }^{* * *} \mathrm{p}\right.$ $=0.0001, * p<0.05)$ b, $\mathrm{S}^{35}$ incorporation in Smg5 KO, Smg5/Eif4a2 ${ }^{\text {PTC }} \mathrm{dKO}$, Smg5/Eif4a2 dKO relative to WT. Cells in ES-DMEM-2i were pulsed with $40 \mu \mathrm{Ci}$ of $\mathrm{S}^{35}$ for 30 min before harvesting. Mean and SD are plotted; $\mathrm{n}=4$ biological replicates. Unpaired t-test was used to calculate $\mathrm{p}$-values $\left({ }^{* * * *} \mathrm{p}<0.0001,{ }^{*} \mathrm{p} \leq\right.$ 0.05). c, $\mathrm{S}^{35}$ incorporation in Smg6 KO, Smg6/Eif4a2 $2^{P T C}$ dKO, Smg6/Eif4a2 dKO relative to WT. Experiment was performed as in $6 \mathrm{~b}$. Mean and SD are plotted, $\mathrm{n}=4$ biological replicates. Unpaired t-test was used to calculate $p$-values $(* * p=0.002, * p \leq 0.05)$. $d$, Transcripts enriched by Eif4a $2^{\mathrm{FL}} \mathrm{RIP}$, detected by RT-qPCR. enrichmentlevels of proteins significantly bound by both Eif4a $2^{\mathrm{FL}}$ and Eif4a2 $2^{\mathrm{PTC}}$ ( 75 proteins, see Figure and red dots represent proteins specifically enriched only in Eif4a2 $2^{\mathrm{FL}}$ co-IP (178 proteins, see Figure 6e). dots represent proteins specifically enriched only in Eif4a2 ${ }^{\text {PTC }}$ co-IP (231 proteins, see Figure 6e). i, 


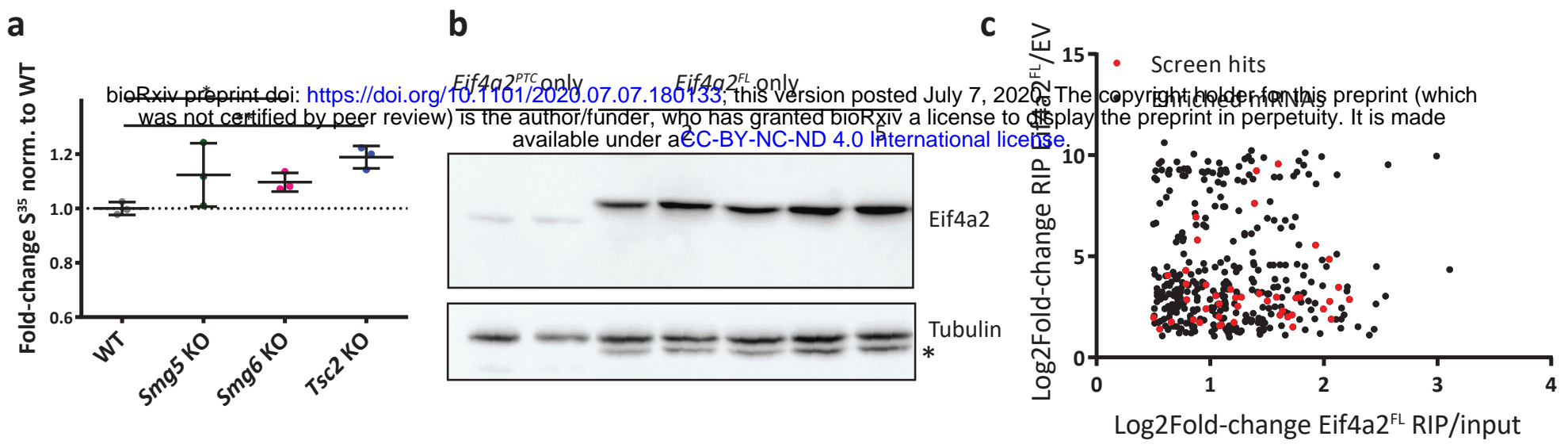

d

e
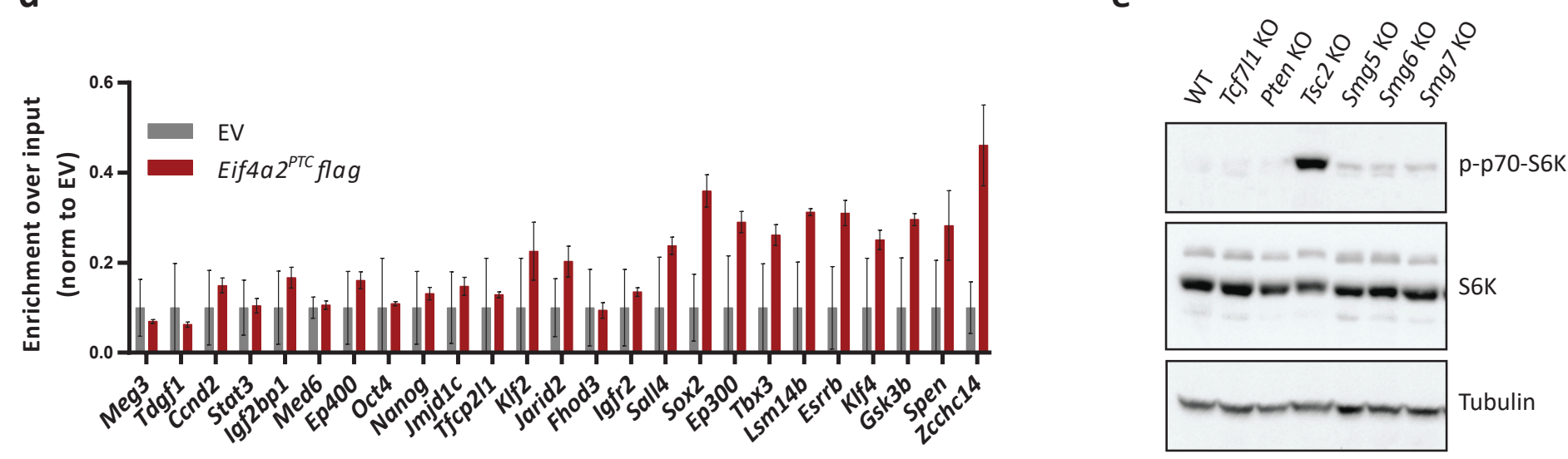


\section{Extended Data Fig. 6 | Eif4a2-mediated differentiation delay is caused by PTC isoform-dependent}

611 regulation of translation a, $\mathrm{S}^{35}$ incorporation levels in NMD KO, TsC2 KO and WT ESCs. Experiment was

612 performed independent from, but as described for Figure 6b. Mean and SD are plotted, $\mathrm{n}=2$ biological

613 replicates. Unpaired t-test was used to calculate $p$-values $\left({ }^{*} p\right.$-value $=0.02,{ }^{* *} p$-value $\left.=0.002\right) . \mathbf{b}$,

614 Western blot analysis for Eif4a2 in Eif4a2 KO cells in which Eif4a2 ${ }^{\mathrm{PTC}}$ or Eif4a2 ${ }^{\mathrm{FL}}$ were re-expressed from

615 transposon vectors. Tubulin was used as a loading control. For Eif4a $2^{\mathrm{FL}}$, only clones 2 and 5 were used

616 for subsequent analysis. * corresponds to Eif4a2 band due to insufficient stripping. c, Significantly

617 enriched transcripts in Eif4a $2^{\mathrm{FL}}$ RIP-Seq (log2fold change Eif4a2 ${ }^{\mathrm{FL}} \mathrm{RIP} /$ input $\geq 0.5, \mathrm{p} \leq 0.05$ and log2fold

618 change Eif4a2 $2^{\mathrm{FL}} \mathrm{RIP} / \mathrm{EV}$ RIP $\left.\geq 1, \mathrm{p} \leq 0.05\right)$. Transcripts of genes with shown function as drivers of the exit

619 from naïve pluripotency ${ }^{6}$ are depicted in red. $d$, Transcripts levels of the indicated genes after Eif4a2 ${ }^{\text {PTC }}$

620 RIP-qPCR. The non-protein coding gene Meg3 was used as negative control. Values were first

621 normalized to EV RIP and then to input. Mean and SD are plotted, $\mathrm{n}=2$ biological replicates. e, Western

622 blot analysis in the indicated cells line in $2 \mathrm{i}$ for mTOR targets. Antibodies used are indicated in the figure.

623 Tubulin was used as loading control. 
gRNAs were designed using http://crisprscan.org. Annealed oligos (Extended Data Table 5) were cloned, coding sequence of the gene of interest was amplified by PCR and cloned into a pCAG-3xFLAG-empty-pgkhph vector ${ }^{5}$ using a BamHI site. The sequence of Eif4a2 $2^{\mathrm{FL}}$ was in vitro synthetized (IDT) and then cloned into the pCAG-3xFLAG-empty-pgk-hph vector. For the mutagenesis of Eif4a2 ${ }^{\text {PTC }}$ the pCAG-3xFLAGEif4a2 ${ }^{\text {PTC }}$-pgk-hph vector was cut using a BamHI and a Sacll site. This generated an Eif4a2 ${ }^{\text {PTC }}$ fragment that was missing the first 17 nucleotides, thus generating an out of frame truncated protein product. The mutated insert was blunt ended and re-cloned into the pCAG-3xFLAG-empty-pgk-hph vector cut with Smal. The correct insertion was verified for all the constructs by restriction digest and Sanger sequencing with the 3xFlag_seq primer (Extended Data Table 6).

Teratoma assay

Paraffin-embedded teratoma tissue blocks were cut on a rotary microtome RM2255 (Leica). The $3 \mu \mathrm{m}$ sections were then stained for hematoxylin and eosin in the automated slide stainer Gemini AS (Histocom) and mounted in Eukitt. Slides were scanned on a VS120 (Olympus) slide scanner.

\section{Cell culture} as parental cell line for all the knockout cells generated in this study. 
streptomycin (Sigma-Aldrich, P4333), $55 \mu \mathrm{M} \beta$-mercaptoethanol (Fisher-Scientific, 21985-023), $2 \mathrm{i}$ (1.5 $\mu \mathrm{M}$ CHIR99021 and $0.5 \mu \mathrm{M}$ PD0325901) and $10 \mathrm{ng} / \mathrm{ml}$ LIF (batch tested, in-house) (ES DMEM-2i medium).

\section{Monolayer differentiation}

For differentiation, ESCs were plated on gelatin-coated plates at a density of $1 \times 10^{4} \mathrm{cells} / \mathrm{cm}^{2}$ in basal medium (N2B27), which is composed by 1:1 ratio of DMEM/F12 (Gibco, 21331020) and Neurobasal medium (Gibco, 21103049) supplemented with 0.5x N2 (homemade), 1x B-27 Serum-Free Supplement (Gibco, 17504-044), 2 mM L-Glutamine (Sigma-Aldrich, G7513), 0.1 mM NEAA (Sigma-Aldrich, M7145), 10 $\mu \mathrm{g} / \mathrm{ml}$ penicillin-streptomycin (Sigma-Aldrich, P4333), $55 \mu \mathrm{M}$ ß-mercaptoethanol (Fisher-Scientific, 21985023) and $2 \mathrm{i}(3 \mu \mathrm{M}$ CHIR99021 and $1 \mu \mathrm{M}$ PD0325901) (N2B27-2i). The following day $2 i$ were withdrawn and cells were differentiated for the indicated time in N2B27.

\section{Commitment assay}

For commitment assay, ESCs were plated on gelatin-coated plates in N2B27-2i medium at a density of $2 \mathrm{x}$ $10^{3}$ cells $/ \mathrm{cm}^{2}$. The following day $2 \mathrm{i}$ were withdrawn. After $48 \mathrm{~h}$ of differentiation medium was changed and selection for Rex1-GFPd2 expressing cells was carried out by adding $5 \mathrm{ng} / \mathrm{ml}$ blasticidin ((BSD) Gibco, R210-01) to the N2B27 medium. The following day medium was changed to N2B27-2i + BSD medium, and medium was refreshed every two days. Alkaline-phosphatase staining was performed after four days in N2B27-2 $i+B S D$ according to the manufacturer's protocol (Sigma-Aldrich, 86R). In brief, cells were fixed using the Citrate-Acetone-Formaldehyde solution. Cells were then stained for 30 min at $4^{\circ} \mathrm{C}$ using a Sodium Nitrite-FRV-Alkaline-Naphthol AS-BI Alkaline solution. Plates were then imaged using an Olympus Cell-Sense microscope (OLYMPUS).

\section{EB differentiation assay}

For embryoid body differentiation, $1 \times 10^{5}$ cells were used and plated in ES DMEM without $2 \mathrm{i}$ and LIF as hanging drops. After two days cells were collected and plated in a $10 \mathrm{~cm}$ petri dish, and medium was 
changed every two days. Cells were harvested every two days and RNA was extracted using the ExtractME kit (LabConsulting, EM09).

\section{Generation of knockout cell lines}

$2 \times 10^{5}$ cells were transfected in a gelatin-coated 6-well plate in ES DMEM-2i using Lipofectamine 2000 (Fisher Scientific, 11668-027). Two pairs of gRNA were used for each gene (1 $\mu \mathrm{g}$ each) together with 0.5 $\mu \mathrm{g}$ of pCAG-dsRed (see full list of gRNAs in Extended Table 6). After $48 \mathrm{~h}$ to enrich for transfected cells, dsRED/GFP double positive cells were sorted using a BD FACS Aria III. Sorted cells were plated at clonal density in ES DMEM-2i. One week after sorting 48 colonies were picked (96 in case of the Smg5/Smg6 dKO cells). Colonies were then trypsinized and half of the cell suspension was plated in a 96-well plate for expansion. The remaining cells were lysed and DNA was extracted for PCR genotyping ${ }^{6}$.

\section{PCR genotyping}

For DNA lysis half of a picked colony was pelleted in a PCR plate at 500g. After two PBS washes, cell pellets were boiled in water at $95^{\circ} \mathrm{C}$ for $5 \mathrm{~min} .3 \mu \mathrm{g} / \mathrm{ml}$ of proteinase $\mathrm{K}$ was added after cooling down the plate and incubated at $65^{\circ} \mathrm{C}$ for $1 \mathrm{~h}$. Reaction was inactivated for $10 \mathrm{~min}$ at $95^{\circ} \mathrm{C}$. To genotype the $\mathrm{KO}$ cells we used a triple-primer strategy. One reverse primer specific for the deletion and one specific for the WT allele were used in combination with a common forward primer (Extended Data Table 6). PCR results were verified by Sanger sequencing and KO was confirmed by western-blot analysis. For genotyping PCR JumpStart RedTaq PCR master mix (Sigma-Aldrich) was used following the manufacturer's protocol for cycling with an annealing temperature of $55^{\circ} \mathrm{C}$ and 35 cycles.

\section{RNAi assay}

$1.5 \times 10^{4}$ cells $/ \mathrm{cm}^{2}$ were transfected in a gelatin-coated 12-well plate in N2B27-2i using DharmaFECT 1 (Fisher Scientific, T-2001). For RNAi assays esiRNA for Eif4a2 (Sigma-Aldrich) and FlexiTube siRNAs for Smg6 and Smg7 (Qiagen) were used. For siRNAs $20 \mathrm{ng}$ siRNAs $/ 4 \times 10^{4}$ cells were used, whereas for esiRNAs 
$200 \mathrm{ng} / 6 \times 10^{4}$ cells were used. The following day, after two PBS washes, medium was changed to N2B27.

\section{Cell cycle analysis}

ESCs were plated in gelatin-coated plates in ES DMEM-2i at a density of $1 \times 10^{4} \mathrm{cells} / \mathrm{cm}^{2}$. After two days cells were harvested and fixed overnight in $70 \%$ ethanol. Cells were then incubated with $100 \mu \mathrm{g} / \mathrm{ml}$ RNase A solution (Qiagen, 19101). 50 mg/liter Propidium lodide solution (Sigma-Aldrich, P4170) was then added to the cells. Cell cycle profiles were recorded on the LSRFortessa flow cytometer (BD bioscience).

\section{Flow Cytometry}

Cells were harvested using $0.25 \%$ trypsin/EDTA and trypsin was neutralized using ES DMEM. Rex1-GFPd2 was measured with LSRFortessa flow cytometer (BD bioscience). High-throughput-measurements were acquired using a 96-well plate HTS unit on the LSRFortessa flow cytometer. Data analysis was performed using flowJo software (BD bioscience).

NMD inhibition assay

707 ESCs were plated in gelatin-coated plates in ES DMEM-2i at a density of $2 \times 10^{4} \mathrm{cells} / \mathrm{cm}^{2}$. The following day cells were treated for $8 \mathrm{~h}$ with either ES DMEM-2i + DMSO or ES DMEM- $2 \mathrm{i}+\mathrm{CHX}(100 \mathrm{ug} / \mathrm{ml}$ (SigmaAldrich, 01810)). RNA was then extracted using ExtractME kit (LabConsulting, EM09) according to the

710 manufacturer's protocol.

\section{Telomere quantification}

712 For telomere quantification genomic DNA was extracted from cells using the Puregene core Kit A (Qiagen).

713 DNA was quantified using PicoGreen Assay for dsDNA (Fisher Scientific, P11496) on a NanoDrop 3300 
Fluorospectrometer (Fisher Scientific). PCR reactions were performed on a CFX384 Touch Real-Time PCR Detection System (BioRad) using telomere specific primers and primers for the single-copy gene 36B4 (Extended Data Table 6). For each primer pair, a standard curve was created with known amounts of DNA to determine primer efficiency. The telomere signal was normalized with the single-copy gene.

\section{RNA analysis}

RNA was extracted using the ExtractMe kit (LabConsulting, EM09) according to the manufacturer's protocol. cDNA was retrotranscribed from $0.3 \mu \mathrm{g}$ to $1 \mu \mathrm{g}$ using the SensiFAST cDNA Synthesis Kit (Bioline, BIO-65054). Real-time PCR was performed on the CFX384 Touch Real-time PCR Detection System (BioRad) using the Sensifast SYBR No Rox-Kit (Bioline, BIO-98020). Expression levels were normalized to either L32 or Actin, as indicated in the figure legends (see full list of primers used for qPCR In Extended Data Table 6). Results are shown as mean and standard deviation.

\section{Protein analysis}

Proteins were extracted using RIPA buffer (Sigma-Aldrich, 20-188) supplemented with Complete Mini EDTA-free Protease inhibitor cocktail (Roche, 04693159001) and PhosSTOP (Phosphatase Inhibitor Cocktail (Roche, 04906845001)). 5\% milk was used for blocking. Primary antibodies were incubated overnight at $4^{\circ} \mathrm{C}$. Washes were performed using PBS-T (Sigma-Aldrich, P4417). Secondary antibodies were incubated for $1 \mathrm{~h}$ at room-temperature. Primary antibodies were used at a dilution of 1:250 for anti-Smg5 (Abcam, ab33033, rabbit), 1:1000 for anti-Smg6 (Abcam, ab87539, rabbit), 1:2000 for anti Smg7 (NovusBio, NBP1-22967, rabbit), 1:1000 for anti-Phosho-Upf1 (Millipore, 07-1016, rabbit), 1:1000 for antiUpf1 (D15G6) (Cell Signaling, 12040S, rabbit), 1:1000 for anti-flag M2 (Sigma-Aldrich, F1804, mouse), 1:1000 for anti-c-Myc (D84C12) (Cell Signaling, 5605T, rabbit), 1:1000 for anti-Tcf7l1 (Fisher Scientific, PA5-40327, rabbit), 1:1000 for anti-Eif4a2 (Abcam, ab31218, rabbit), 1:1000 for anti-Eif4a1 (Cell Signaling, 2490T, rabbit), 1:1000 for anti-Nanog (NovusBio, NB100-58842, rabbit), 1:1000 for anti-Esrrb (RND 
systems, PP-H6705-00, mouse), 1:1000 for anti-p-p70-S6K (Thr389) (108D2) (Cell Signaling, 9234T, rabbit), 1:1000 for anti-S6K (49D7) (Cell Signaling, 2708T, rabbit), 1:5000 for anti-Tubulin (Sigma-Aldrich, T8203, mouse), 1:5000 for anti-Gapdh (Sigma-Aldrich, G8795, mouse) and 1:5000 for anti-Vinculin (E1E9V) (CellSignaling, 13901T, rabbit). Secondary antibodies were used at a dilution of 1:10000 for anti-rabbit IgG (Amersham, NA934), 1:15000 for goat anti-mouse IgG (Santa Cruz, sc-2064). Chemiluminescence signal from antibody binding was detected using ECL Select detection kit (GE healthcare, GERPN2235) with a ChemiDoc system (BioRad).

\section{Immunoprecipitation}

Cells were plated in ES DMEM-2i, after two days they were harvested in IP lysis buffer (10 mM Tris Base, $10 \mathrm{mM} \mathrm{NaCl}, 2 \mathrm{mM}$ EDTA, 0,5\% Triton X-100) supplemented with Complete Mini EDTA-free Protease inhibitor cocktail (Roche, 04693159001) and PhosSTOP (Phosphatase Inhibitor Cocktail (Roche, 04906845001)). $1 \mathrm{mg}$ of lysate was used for the immunoprecipitation. Briefly, Dynabeads (Fisher Scientific, 10004D) were coated with $5 \mu \mathrm{g}$ of anti-flag M2 antibody (Sigma-Aldrich, F1804, mouse) for $1 \mathrm{~h}$. In case of RNA-free IP, lysates were treated with $50 \mathrm{U} / \mathrm{ml}$ Cyanase (Süd-Laborbedarf SLG, CY1000) and 0.5 $\mathrm{ul} / \mathrm{ml}$ of $2 \mathrm{M} \mathrm{MnSO}_{4}$ and incubated on ice for $10 \mathrm{~min}$. Lysates were then cleared out by centrifugation at $16000 \mathrm{~g}$ for $15 \mathrm{~min}$ at $4^{\circ} \mathrm{C}, 1 \%$ of each lysate was kept as input. $\mathrm{NaCl}$ was added to a final concentration of $150 \mathrm{mM}$. Dynabeads were washed three times with wash buffer (137 mM NaCl, $20 \mathrm{mM}$ Tris Base, 0,5\% (v/v) Tergitol-type NP-40) and one time with lysis buffer and then were incubated with the lysates for $3 \mathrm{~h}$ at $4^{\circ} \mathrm{C}$. Dynabeads were washed three times with wash buffer supplemented with Complete Mini EDTAfree Protease inhibitor cocktail (Roche, 04693159001) and PhosSTOP (Phosphatase Inhibitor Cocktail (Roche, 04906845001)). Samples were eluted in 2X sample buffer.

For the immunoprecipitation coupled with mass-spec cells were plated in ES DMEM-2i, the following day they were treated with $1 \mu \mathrm{M}$ epoxomicin (Gentaur, 607-A2606) for $3 \mathrm{~h}$. Cells were harvested and lysed in 
IP lysis buffer. $1 \mathrm{mg}$ of lysate was used for the immunoprecipitation. Immunoprecipitation protocol was followed (see above) with the addition of the cross-linking of the anti-flag M2 antibody to the Dynabeads using dimethyl pimelimidate (DMP) (Sigma-Aldrich, D-8388). Briefly, after antibody coupling, Dynabeads were washed three times with $200 \mathrm{mM}$ Sodium Borate $(\mathrm{pH}=9)$ and then incubated for 30 min with DMPSodium Borate solution. They were washed with the following buffers: three times with $250 \mathrm{mM}$ Tris $(\mathrm{pH}=8.0)$, two times with $100 \mathrm{mM}$ glycine $(\mathrm{pH}=2)$, three times with TBS-T and one time with lysis buffer. Beads were then incubated with the lysates for $2 \mathrm{~h}$ at $4^{\circ} \mathrm{C}$. Five washes were performed with wash buffer (137 mM NaCl, 20 mM Tris Base) and samples were submitted for mass-spec.

\section{Sample preparation for mass spectrometry analysis}

Beads with cross-linked antibody were transferred to new tubes and resuspended in $30 \mu \mathrm{L}$ of $2 \mathrm{M}$ urea in $50 \mathrm{mM}$ ammonium bicarbonate $(A B C)$. Disulfide bonds were reduced with $10 \mathrm{mM}$ dithiothreitol for 30 min at room temperature before adding $25 \mathrm{mM}$ iodoacetamide and incubating for $15 \mathrm{~min}$ at room temperature in the dark. Remaining iodoacetamide was quenched by adding $5 \mathrm{mM}$ DTT and the proteins were digested with $150 \mathrm{ng}$ trypsin (Trypsin Gold, Promega) at room temperature for $90 \mathrm{~min}$. The supernatant was transferred to a new tube, the beads were washed with another $30 \mu \mathrm{L}$ of $2 \mathrm{M}$ urea in 50 $\mathrm{mM} A B C$ and the wash combined with the supernatant. After diluting to $1 \mathrm{M}$ urea with $50 \mathrm{mM} \mathrm{ABC}$, additional $150 \mathrm{ng}$ trypsin were added and incubated overnight at $37^{\circ} \mathrm{C}$ in the dark. The digest was stopped by addition of trifluoroacetic acid (TFA) to a final concentration of $0.5 \%$, and the peptides were desalted using C18 Stagetips ${ }^{47}$. Peptides were separated on an Ultimate 3000 RSLC nano-flow chromatography system (Thermo-Fisher), using a pre-column for sample loading (Acclaim PepMap C18, $2 \mathrm{~cm} \times 0.1 \mathrm{~mm}, 5$ $\mu \mathrm{m}$, Thermo-Fisher), and a C18 analytical column (Acclaim PepMap C18, $50 \mathrm{~cm} \times 0.75 \mathrm{~mm}, 2 \mu \mathrm{m}$, ThermoFisher), applying a segmented linear gradient from $2 \%$ to $35 \%$ and finally $80 \%$ solvent B ( $80 \%$ acetonitrile, $0.1 \%$ formic acid; solvent $\mathrm{A} 0.1 \%$ formic acid) at a flow rate of $230 \mathrm{~nL} / \mathrm{min}$ over $120 \mathrm{~min}$. Eluting peptides were analyzed on a Q Exactive HF-X Orbitrap mass spectrometer (Thermo Fisher), which was coupled to 
the column with a customized nano-spray EASY-Spray ion-source (Thermo-Fisher) using coated emitter tips (New Objective).

\section{mRNA half-life measurement:}

ESCs were plated in N2B27-2i at a density of $20 \times 10^{4}$ cells $/ \mathrm{cm}^{2}$. The day after medium was changed to N2B27-2i + $100 \mu \mathrm{M}$ 4SU (Carbosynth, NT06186) and they were cultured in this condition for $12 \mathrm{~h}$. After a PBS wash, cells were incubated with N2B27-2i medium + 10 mM Uridine (Sigma-Aldrich, U6381) for $3 \mathrm{~h}$. RNA extraction was carried out using Trizol (Fisher-Scientific, 10296-010) following the manufacturer's instruction with the addition of $0.1 \mathrm{mM}$ of DTT during the isopropanol precipitation. RNA was resuspended in $1 \mathrm{mM}$ DTT. $5 \mu \mathrm{g}$ of RNA were treated with $10 \mathrm{mM}$ iodoacetamide (Sigma-Aldrich, 11149) followed by ethanol precipitation ${ }^{29} .2 \mathrm{ng}$ of RNA were used for library prep. Libraries were prepared using the QuantSeq 3'mRNA-seq Library Prep Kit for Illumina FWD (Lexogen, R3142) and were analyzed on a HiSeqV4 SR100.

\section{RNA-Immunoprecipitation}

$3 \times 10^{6}$ cells were plated in ES-DMEM. After two days cells were harvested and lysed using the MAGNARIP kit (Millipore 17-700) according to the manufacturer's protocol. Briefly, cells were lysed by a freeze and thaw cycle and lysates were stored at $-80^{\circ} \mathrm{C}$. Immunoprecipitations were performed at $4^{\circ} \mathrm{C}$ for $3 \mathrm{~h}$ using $5 \mu \mathrm{g}$ of an anti-FLAG M2 antibody (Sigma-Aldrich, F1804, mouse). For RIP-Seq, libraries were prepared using the QuantSeq 3'mRNA-seq Library Prep Kit for Illumina FWD (Lexogen, R3142) and analyzed on a HiSeqV4 SR50. For RIP-qPCR, reverse transcription and qPCR were carried out as above. Relative binding to input and empty vector control was calculated. Error bars show the standard deviation between technical duplicates (Eif4a2 ${ }^{\mathrm{FL}} \mathrm{RIP}$ ) and between biological duplicates (Empty vector and Eif4a2 ${ }^{\mathrm{PTC}}$ RIP). 


\section{Translation rates measurement with $\mathbf{S}^{35}$ incorporation}

808

809

810

811

812

813

ESCs were cultured in ES DMEM and $33 \mu \mathrm{Ci} \mathrm{S}^{35}$ (Hartmann, IS-103) was added to the culture media for 30 min. Cells were lysed in RIPA buffer (Sigma-Aldrich, 20-188) supplemented with Complete Mini EDTA-free Protease inhibitor cocktail (Roche, 04693159001 ) as described above. Protein extracts were then spotted on a nitrocellulose membrane. Membranes were stained with Ponceau. The membranes were wrapped in saran wrap and exposed to a BAS Storage Phosphor Screen (GE Healthcare). After two days signal was acquired using a Typhoon scanner (GE Healthcare). Radioactive signal was quantified using Fiji. $\mathrm{S}^{35}$ signal was normalized to ponceau staining. Results are shown as mean and standard deviation.

\section{Translation rates measurement with OPP incorporation}

ESCs were cultured in N2B27 2i medium, the following day 2i were withdrawn. After $12 \mathrm{~h}$ of differentiation the medium was changed to N2B27 supplemented with OPP (Fisher Scientific) for a 20 s pulse. Cells were then washed two times in N2B27 medium and then N2B27 medium was added for a 10 min chase. Cells were then harvested. Fixation, permeabilization and Click-IT reaction were performed using the Click-iT Plus OPP Alexa Fluor 647 Protein Synthesis Assay Kit (Fisher Scientific, C10458), according to the manufacturer's protocol. Fluorescent signal was acquired with LSRFortessa flow cytometer (BD bioscience). Median OPP-647 signal was used for quantification. Results are shown as mean and standard deviation. 
Data analysis:

\section{RNA-seq differential analysis}

RNA-seq samples and analysis used in this study were taken from Lackner and colleagues ${ }^{6}$

\section{GO enrichment analysis}

GO analysis annotation were taken from the R package org.Mm.eg.db (Version 3.4.0). For the enrichment of GO Terms in the different gene lists we only considered terms with 5 to 500 genes assigned to them. Significance of the enrichment was determined using Fisher's exact test with all expressed genes as background (Lackner et al., 2020). GO terms that did not differ in more than 5 genes were clustered and one representative term for each cluster was defined (using the $\mathrm{R}$ base function hclust on the L1-distance of the binary membership matrix). The representative term for each cluster was the term with the least total annotated genes. Multiple hypothesis testing was executed using the Benjamini-Hochberg method on all representative terms to calculate adjusted $p$-values. background (no 4SU treatment) was subtracted to $\mathrm{T}>\mathrm{C}$ conversion rates of transcripts with $\mathrm{CPM} \geq 2$. Half-

844 lives were calculated by single exponential fit to a decay model ${ }^{29}$.

mRNA half-lives differential analysis

T>C conversion rates were used to model the half-life changes. Background (no 4SU treatment) was

847 subtracted from $\mathrm{T}>\mathrm{C}$ conversion rates and then $\mathrm{T}>\mathrm{C}$ conversion rates of transcripts with $\mathrm{CPM} \geq 2$ were considered for the differential analysis. Differential analysis to quantify the change in $\mathrm{T}>\mathrm{C}$ conversion rates rates $\sim$ time ${ }^{*}$ genotype. In brief, firstly the $\mathrm{T}>\mathrm{C}$ conversion rates after $3 \mathrm{~h}$ of Uridine chase were 
normalized to the T>C conversion rates of the 4SU pulse. Secondly, the beta regression model quantified the difference between T>C conversion rates of WT and NMD KO cells after $3 \mathrm{~h}$ of Uridine chase. $\mathrm{p}$-values were determined by a partial Wald test. To determine transcripts with a significant change in expression and half-life we considered the 516 upregulated transcripts from Figure 3a. We could calculate a half-life change for 250 out of 516 transcripts. To obtain direct targets, we postulated that upregulated transcripts should have a concomitant increase in half-life (half-life change $\geq 0.2$ ). Finally, to select NMD targets relevant in ESCs out of these 136 transcripts we isolated the 57 that were upregulated in expression according to the graded phenotypes.

\section{Exon conservation}

Pairwise whole genome alignments of mouse ( $\mathrm{GRCm} 38 / \mathrm{mm} 10)$ against dog (canFam3), rat (RGSC6.0/rn6) and human (GRCh38/hg38) from UCSC (http://hgdownload.cse.ucsc.edu/downloads.html) were used for the analysis. With the Ensembl annotation of the mouse genome we extracted the respective regions of the genes (introns, exons and UTRs) and calculated their sequence identities compared to the other organism in the pairwise alignment as (number of matching nucleotide pairs in the alignment)*100/length(alignment). As gene region annotation can be ambiguous due to variations in transcript splicing, we used the following definitions. Everything that is annotated as coding sequence in any protein coding transcript we count as coding exon. UTR is everything that is annotated as UTR in any protein coding transcript except if it is also annotated as coding sequence in which case it is counted as coding exon. Regions not covered by the above definitions are counted as introns.

\section{RIP-Seq differential analysis}

Quality control of the transcripts from input and RIP samples was performed using fastQC. Reads were trimmed using bbduk (Version 38.57). Reads were mapped to the mm10 mouse reference genome with STAR (Version 2.5.3a). Afterwards indexing was performed using samtool (Version 1.5) and reads in 
transcripts were counted with HTSeq-count (Version 0.11.2). Transcripts that had cpm more or equal to one in all the input samples were considered for the differential analysis. Differential analysis was performed using DESeq2 (Version 1.24.0). To identify significantly enriched transcripts in Eif4a2 $2^{\mathrm{FL}}$ RIP we considered only the 1,231 transcripts with $\mathrm{cpm} \geq 30$ in Eif4a $2^{\mathrm{FL}} \mathrm{RIP}$ and either log-fold-change Eif4a2 $2^{\mathrm{FL}}$ input / Eif4a2 $2^{\mathrm{FL}} \mathrm{RIP} \geq 1$ and $\leq-1$ ( $p$-value $\leq 0.05$ ) or log-fold-change Eif4a $2^{\mathrm{FL}} \mathrm{RIP} / \mathrm{EV} \mathrm{RIP} \geq 1$ and $\leq-1$ ( $p$-value $\leq 0.05$ ). Out of those we considered as enriched only the transcripts that had Eif4a2 $2^{\mathrm{FL}}$ input / Eif4a $2^{\mathrm{FL}} \mathrm{RIP} \geq 0.5$ and log-fold-change Eif4a2 $2^{\mathrm{FL}} \mathrm{RIP} / \mathrm{EV}$ RIP $\geq 1$ (362 transcripts).

\section{Mass spectrometry data acquisition and analysis}

The mass spectrometer was operated in data-dependent acquisition mode (DDA), survey scans were obtained in a mass range of $375-1500 \mathrm{~m} / \mathrm{z}$ with lock mass activated, at a resolution of $120 \mathrm{k}$ at $200 \mathrm{~m} / \mathrm{z}$ and an AGC target value of 3E6. The 8 most intense ions were selected with an isolation width of $1.6 \mathrm{~m} / \mathrm{z}$, fragmented in the HCD cell at $28 \%$ collision energy and the spectra recorded for max. $250 \mathrm{~ms}$ at a target value of $1 \mathrm{E} 5$ and a resolution of $30 \mathrm{k}$. Peptides with a charge of +1 or $>+7$ were excluded from fragmentation, the peptide match feature was set to preferred, the exclude isotope feature was enabled, and selected precursors were dynamically excluded from repeated sampling for $30 \mathrm{~s}$.

Raw data were processed using the MaxQuant software package (version 1.6.0.16, ${ }^{48}$ ) and the Uniprot mouse reference proteome (January 2019, www.uniprot.org), target sequences, as well as a database of most common contaminants. The search was performed with full trypsin specificity and a maximum of two missed cleavages at a protein and peptide spectrum match false discovery rate of $1 \%$. Carbamidomethylation of cysteine residues were set as fixed, oxidation of methionine and $\mathrm{N}$-terminal acetylation as variable modifications. For label-free quantification the "match between runs" feature and the LFQ function were activated - all other parameters were left at default. 
MaxQuant output tables were further processed in R (R Core Team, 2018, https://www.R-project.org/).

897 Reverse database identifications, contaminant proteins, protein groups identified only by a modified peptide, protein groups with less than three quantitative values in one experimental group, and protein groups with less than 2 razor peptides were removed for further analysis. Due to differences in overall contaminant levels between samples, LFQ values were re-normalized using the sample median of the "background" protein subset (as identified in controls). Missing values were replaced by randomly drawing data points from a normal distribution modeled on the whole dataset (data mean shifted by -1.8

903 standard deviations, width of distribution of 0.3 standard deviations). Differences between groups were 904 statistically evaluated using the LIMMA package ${ }^{49}$ at 5\% FDR (Benjamini-Hochberg). The mass spectrometry proteomics data have been deposited to the ProteomeXchange Consortium via the PRIDE

906 partner repository ${ }^{50}$ under the following accession number PXD019588. NGS data have been deposited 907 on GEO (https://www.ncbi.nlm.nih.gov/geo/query/acc.cgi?acc=GSE153457, reviewer token: uvgImauwnfufpkp) 


\section{REFERENCES}

1. Dunn, S.J., Martello, G., Yordanov, B., Emmott, S. \& Smith, A.G. Defining an essential transcription factor program for naive pluripotency. Science 344, 1156-1160 (2014).

2. Young, R.A. Control of the embryonic stem cell state. Cell 144, 940-954 (2011).

3. Boroviak, T., Loos, R., Bertone, P., Smith, A. \& Nichols, J. The ability of inner-cell-mass cells to self-renew as embryonic stem cells is acquired following epiblast specification. Nature cell biology 16, 516-528 (2014).

4. Wray, J. et al. Inhibition of glycogen synthase kinase-3 alleviates Tcf3 repression of the pluripotency network and increases embryonic stem cell resistance to differentiation. Nature cell biology 13, 838-845 (2011).

5. Betschinger, J. et al. Exit from pluripotency is gated by intracellular redistribution of the bHLH transcription factor Tfe3. Cell 153, 335-347 (2013).

6. Lackner, A. et al. Cooperative molecular networks drive a mammalian cell state transition. bioRxiv, 2020.2003.2023.000109 (2020).

7. Mulas, C., Kalkan, T. \& Smith, A. NODAL Secures Pluripotency upon Embryonic Stem Cell Progression from the Ground State. Stem Cell Reports 9, 77-91 (2017).

8. Kalkan, T. et al. Tracking the embryonic stem cell transition from ground state pluripotency. Development (Cambridge, England) 144, 1221-1234 (2017).

9. Li, Q.V., Rosen, B.P. \& Huangfu, D. Decoding pluripotency: Genetic screens to interrogate the acquisition, maintenance, and exit of pluripotency. WIREs Systems Biology and Medicine 12, e1464 (2020).

10. Batista, Pedro J. et al. m6A RNA Modification Controls Cell Fate Transition in Mammalian Embryonic Stem Cells. Cell stem cell 15, 707-719 (2014).

11. Geula, S. et al. $m<$ sup $>6</$ sup $>A$ mRNA methylation facilitates resolution of naïve pluripotency toward differentiation. Science 347, 1002-1006 (2015).

12. Leeb, M., Dietmann, S., Paramor, M., Niwa, H. \& Smith, A. Genetic exploration of the exit from self-renewal using haploid embryonic stem cells. Cell Stem Cell 14, 385-393 (2014).

13. Li, T. et al. Smg6/Est1 licenses embryonic stem cell differentiation via nonsense-mediated mRNA decay. The EMBO journal 34, 1630-1647 (2015).

14. Lou, C.-H. et al. Nonsense-Mediated RNA Decay Influences Human Embryonic Stem Cell Fate. Stem cell reports 6, 844-857 (2016). 
15. Kurosaki, T., Popp, M.W. \& Maquat, L.E. Quality and quantity control of gene expression by nonsense-mediated mRNA decay. Nature Reviews Molecular Cell Biology 20, 406-420 (2019).

16. Mendell, J.T., Sharifi, N.A., Meyers, J.L., Martinez-Murillo, F. \& Dietz, H.C. Nonsense surveillance regulates expression of diverse classes of mammalian transcripts and mutes genomic noise. Nature Genetics 36, 1073-1078 (2004).

17. Wittmann, J., Hol, E.M. \& Jäck, H.-M. hUPF2 silencing identifies physiologic substrates of mammalian nonsense-mediated mRNA decay. Mol Cell Biol 26, 1272-1287 (2006).

18. Tani, H. et al. Identification of hundreds of novel UPF1 target transcripts by direct determination of whole transcriptome stability. RNA Biology 9, 1370-1379 (2012).

19. Colombo, M., Karousis, E.D., Bourquin, J., Bruggmann, R. \& Mühlemann, O. Transcriptome-wide identification of NMD-targeted human mRNAs reveals extensive redundancy between SMG6and SMG7-mediated degradation pathways. RNA 23, 189-201 (2017).

20. Reichenbach, P. et al. A Human Homolog of Yeast Est1 Associates with Telomerase and Uncaps Chromosome Ends When Overexpressed. Current Biology 13, 568-574 (2003).

21. Azzalin, C.M., Reichenbach, P., Khoriauli, L., Giulotto, E. \& Lingner, J. Telomeric RepeatContaining RNA and RNA Surveillance Factors at Mammalian Chromosome Ends. Science 318, 798 (2007).

22. Chawla, R. \& Azzalin, C.M. The telomeric transcriptome and SMG proteins at the crossroads. Cytogenetic and Genome Research 122, 194-201 (2008).

23. Li, M. et al. Genome-wide CRISPR-KO Screen Uncovers mTORC1-Mediated Gsk3 Regulation in Naive Pluripotency Maintenance and Dissolution. Cell Rep 24, 489-502 (2018).

24. Jonas, S., Weichenrieder, O. \& Izaurralde, E. An unusual arrangement of two 14-3-3-like domains in the SMG5-SMG7 heterodimer is required for efficient nonsense-mediated mRNA decay. Genes Dev 27, 211-225 (2013).

25. Okada-Katsuhata, Y. et al. N- and C-terminal Upf1 phosphorylations create binding platforms for SMG-6 and SMG-5:SMG-7 during NMD. Nucleic acids research 40, 1251-1266 (2012).

26. Ohnishi, T. et al. Phosphorylation of hUPF1 Induces Formation of mRNA Surveillance Complexes Containing hSMG-5 and hSMG-7. Molecular Cell 12, 1187-1200 (2003).

27. Yepiskoposyan, H., Aeschimann, F., Nilsson, D., Okoniewski, M. \& Mühlemann, O. Autoregulation of the nonsense-mediated mRNA decay pathway in human cells. RNA 17, 21082118 (2011). 
$971 \quad 28 . \quad H u a n g$, L. et al. RNA homeostasis governed by cell type-specific and branched feedback loops acting on NMD. Molecular cell 43, 950-961 (2011).

29. Herzog, V.A. et al. Thiol-linked alkylation of RNA to assess expression dynamics. Nature Methods 14, 1198-1204 (2017).

30. Yoder-Hill, J., Pause, A., Sonenberg, N. \& Merrick, W.C. The p46 subunit of eukaryotic initiation factor (elF)-4F exchanges with elF-4A. Journal of Biological Chemistry 268, 5566-5573 (1993).

31. Li, W., Belsham, G.J. \& Proud, C.G. Eukaryotic Initiation Factors 4A (elF4A) and 4G (elF4G) Mutually Interact in a 1:1 Ratio in Vivo. Journal of Biological Chemistry 276, 29111-29115 (2001).

32. Grabole, N. et al. Genomic analysis of the molecular neuropathology of tuberous sclerosis using a human stem cell model. Genome medicine 8, 94 (2016).

33. Keene, J.D., Komisarow, J.M. \& Friedersdorf, M.B. RIP-Chip: the isolation and identification of mRNAs, microRNAs and protein components of ribonucleoprotein complexes from cell extracts. Nature Protocols 1, 302-307 (2006).

34. Meng, L. et al. Epoxomicin, a potent and selective proteasome inhibitor, exhibits \&lt;em\&gt;in vivo\&lt;/em\&gt; antiinflammatory activity. Proceedings of the National Academy of Sciences 96, 10403 (1999).

35. Schweingruber, C., Soffientini, P., Ruepp, M.D., Bachi, A. \& Mühlemann, O. Identification of Interactions in the NMD Complex Using Proximity-Dependent Biotinylation (BiolD). PLoS One 11, e0150239 (2016).

36. Jang, H. et al. $<$ em $>0</ e m>-$ GlcNAc Regulates Pluripotency and Reprogramming by Directly Acting on Core Components of the Pluripotency Network. Cell stem cell 11, 62-74 (2012).

37. Bornelöv, S., Selmi, T., Flad, S., Dietmann, S. \& Frye, M. Codon usage optimization in pluripotent embryonic stem cells. Genome Biology 20, 119 (2019).

38. Di Stefano, B. et al. The RNA Helicase DDX6 Controls Cellular Plasticity by Modulating P-Body Homeostasis. Cell stem cell 25, 622-638.e613 (2019).

39. Bulut-Karslioglu, A. et al. The Transcriptionally Permissive Chromatin State of Embryonic Stem Cells Is Acutely Tuned to Translational Output. Cell stem cell 22, 369-383.e368 (2018).

40. Corsini, N.S. et al. Coordinated Control of mRNA and rRNA Processing Controls Embryonic Stem Cell Pluripotency and Differentiation. Cell stem cell 22, 543-558.e512 (2018).

41. You, K.T., Park, J. \& Kim, V.N. Role of the small subunit processome in the maintenance of pluripotent stem cells. Genes \& development 29, 2004-2009 (2015). 
1002

1003

1004

1005

1006

1007

1008

1009

1010

1011

1012

1013

1014

1015

1016

1017

1018

1019

1020

1021

1022

42. Shen, R., Weng, C., Yu, J. \& Xie, T. elF4A controls germline stem cell self-renewal by directly inhibiting BAM function in the Drosophila ovary. Proc Natl Acad Sci U S A 106, 11623-11628 (2009).

43. Tuck, A.C. et al. Mammalian RNA Decay Pathways Are Highly Specialized and Widely Linked to Translation. Molecular Cell (2020).

44. Holmes, Z.E. et al. The Sox2 transcription factor binds RNA. Nature Communications 11, 1805 (2020).

45. Gao, Z. et al. Determination of protein interactome of transcription factor Sox2 in embryonic stem cells engineered for inducible expression of four reprogramming factors. The Journal of biological chemistry 287, 11384-11397 (2012).

46. Mali, P. et al. RNA-guided human genome engineering via Cas9. Science 339, 823-826 (2013).

47. Rappsilber, J., Mann, M. \& Ishihama, Y. Protocol for micro-purification, enrichment, prefractionation and storage of peptides for proteomics using StageTips. Nature Protocols 2, 18961906 (2007).

48. Tyanova, S., Temu, T. \& Cox, J. The MaxQuant computational platform for mass spectrometrybased shotgun proteomics. Nature Protocols 11, 2301-2319 (2016).

49. Ritchie, M.E. et al. limma powers differential expression analyses for RNA-sequencing and microarray studies. Nucleic Acids Research 43, e47-e47 (2015).

50. Perez-Riverol, Y. et al. The PRIDE database and related tools and resources in 2019: improving support for quantification data. Nucleic Acids Research 47, D442-D450 (2018). 